\title{
On a stronger Lazer-McKenna conjecture for Ambrosetti-Prodi type problems
}

\author{
JUNCHENG WeI AND SHUSEN YAN
}

\begin{abstract}
We consider an elliptic problem of Ambrosetti-Prodi type involving critical Sobolev exponent on a bounded smooth domain. We show that if the domain has some symmetry, the problem has infinitely many (distinct) solutions whose energy approach to infinity even for a fixed parameter, thereby obtaining a stronger result than the Lazer-McKenna conjecture.
\end{abstract}

Mathematics Subject Classification (2010): 35J65 (primary); 35B38, 47H15 (secondary).

\section{Introduction}

Elliptic problems of Ambrosetti-Prodi type have the following form:

$$
\begin{cases}-\Delta u=g(u)-\bar{s} \varphi_{1}(x), & \text { in } \Omega, \\ u=0, & \text { on } \partial \Omega,\end{cases}
$$

where $g(t)$ satisfies $\lim _{t \rightarrow-\infty} \frac{g(t)}{t}=v<\lambda_{1}, \lim _{t \rightarrow+\infty} \frac{g(t)}{t}=\mu>\lambda_{1}, \lambda_{1}$ is the first eigenvalue of $-\Delta$ with Dirichlet boundary condition and $\varphi_{1}>0$ is the first eigenfunction. Here $\mu=+\infty$ and $v=-\infty$ are allowed. It is well-known that the location of $\mu, v$ with respect to the spectrum of $\left(-\Delta, H_{0}^{1}(\Omega)\right)$ plays an important role in the multiplicity of solutions for problem (1.1). See for example $[3,8,9,18-20,23-26,31-34]$. In the early 1980s, Lazer and McKenna conjectured that if $\mu=+\infty$ and $g(t)$ does not grow too fast at infinity, (1.1) has an unbounded number of solutions as $\bar{s} \rightarrow+\infty$. See [24].

In this paper, we will consider the following special case:

$$
\begin{cases}-\Delta u=u_{+}^{2^{*}-1}+\lambda u-\bar{s} \varphi_{1}, & \text { in } \Omega, \\ u=0, & \text { on } \partial \Omega,\end{cases}
$$

The research of the first author was partially supported by a research grant from GRF of Hong Kong and a Focused Research Scheme of CUHK.

Received December 10, 2008; accepted in revised form May 28, 2009. 
where $\Omega$ is a bounded domain in $\mathbb{R}^{N}$ with $C^{2}$ boundary, $N \geq 3, \lambda<\lambda_{1}, \bar{s}>0$, $u_{+}=\max (u, 0)$ and $2^{*}=2 N /(N-2)$.

It is easy to see that (1.2) has a negative solution

$$
\underline{u}_{\bar{s}}=-\frac{\bar{s}}{\lambda_{1}-\lambda} \varphi_{1},
$$

if $\lambda<\lambda_{1}$. Moreover, if $\underline{u}_{\bar{s}}+u$ is a solution of (1.2), then $u$ satisfies

$$
\begin{cases}-\Delta u=\left(u-s \varphi_{1}\right)_{+}^{2^{*}-1}+\lambda u, & \text { in } \Omega, \\ u=0, & \text { on } \partial \Omega,\end{cases}
$$

where $s=\frac{\bar{s}}{\lambda_{1}-\lambda}>0$.

Let us recall some recent results on the Lazer-McKenna conjecture related to (1.3). Firstly, Dancer and the second author proved in $[12,13]$ that for $N \geq 2$ and $\lambda \in\left(-\infty, \lambda_{1}\right)$, the Lazer-McKenna conjecture is true if the critical exponent in (1.3) is replaced by sub-critical one. In the critical case, it was proved in $[27,28,36]$ that if $N \geq 6$ and $\lambda \in\left(0, \lambda_{1}\right)$, then (1.3) has unbounded number of solutions as $s \rightarrow+\infty$. The solutions constructed for (1.3) concentrate either at the maximum points of the first eigenfunction [27], or at some boundary points of the domain [36] as $s \rightarrow+\infty$. On the other hand, Druet proves in [21] that the conditions $N \geq 6$ and $\lambda \in\left(0, \lambda_{1}\right)$ are necessary for the existence of the peak-solutions constructed in [27,36]. More precisely, the result in [21] states that if $N=3,4,5$, or $N \geq 6$ and $\lambda \leq 0$, then (1.3) has no solution $u_{s}$, such that the energy of $u_{s}$ is bounded as $s \rightarrow+\infty$. This result suggests that it is more difficult to find solutions for (1.3) in the lower dimensional cases $N=3,4,5$, or in the case $\lambda \leq 0$ and $N \geq 6$.

Note that all the results just mentioned state that (1.3) has more and more solutions as the parameter $s \rightarrow+\infty$. But for fixed $s>0$, it is hard to estimate how many solutions (1.3) has. (In the critical case, for fixed $s$, it is even unknown if there is a solution.)

In this paper, we will deal with (1.3) in the lower dimensional cases $N=$ $4,5,6$, or $N \geq 7$ and $\lambda \leq 0$, assuming that the domain $\Omega$ satisfies the following symmetry condition:

(S1): If $x=\left(x_{1}, \cdots, x_{N}\right) \in \Omega$, then, for any $\theta \in[0,2 \pi],\left(r \cos \theta, r \sin \theta, x_{3}, \cdots, x_{N}\right) \in \Omega$, where $r=$ $\sqrt{x_{1}^{2}+x_{2}^{2}}$;

(S2): If $x=\left(x_{1}, \cdots, x_{N}\right) \in \Omega$, then, for any $3 \leq i \leq N,\left(x_{1}, x_{2}, x_{3}, \cdots,-x_{i}, \cdots, x_{N}\right) \in \Omega$. 
The main result of this paper is the following:

Theorem 1.1. Suppose that $\Omega$ satisfies $(S 1)$ and $(S 2)$. Assume that one of the following conditions holds:

(i) $N=4,5, \lambda<\lambda_{1}$ and $s>0$;

(ii) $N=6, \lambda<\lambda_{1}$ and $s>|\lambda| s_{0}$ for some $s_{0}>0$, which depends on $\Omega$ only;

(iii) $N \geq 7, \lambda=0$ and $s>0$.

Then, (1.3) has infinitely many distinct solutions whose energy can approach to infinity.

The result in Theorem 1.1 is stronger than the Lazer-McKenna conjecture. Note that in Theorem 1.1, the constant $s$ is fixed. In fact, all the parameters are fixed. This gives a striking contrast to the results in [27,36], where $s$ is regarded as a parameter which needs to tend to infinity in order to obtain the results there. As far as the authors know, this seems to be the first such result for Ambrosetti-Prodi type problems. We believe Theorem 1.1 should be true in any general domain and hence we pose the following stronger Lazer-McKenna conjecture:

Stronger Lazer-McKenna Conjecture: Let $s$ be fixed and $\lambda<\lambda_{1}$. Then problem (1.3) has infinitely many solutions.

We are not able to obtain similar result for the cases $N=3$, and $N \geq 7$ and $\lambda<0$. But we have the following weaker result for $N \geq 7$ and $\lambda<0$, which gives a positive answer to the Lazer-McKenna conjecture in this case:

Theorem 1.2. Suppose that $\Omega$ satisfies $(S 1)$ and $(S 2)$, and $N \geq 7, \lambda<\lambda_{1}$. Then, the number of distinct solutions for (1.3) is unbounded as $s \rightarrow+\infty$.

Problem (1.3) is a bit delicate in the case $N=3$. When $s=0$, Brezis and Nirenberg [7] proved that (1.3) has a least energy solution if $\lambda \in\left(0, \lambda_{1}\right)$, while for $N=3$, this result holds only if $\lambda \in\left(\lambda^{*}, \lambda_{1}\right)$ for some $\lambda^{*}>0$ (if $\Omega$ is a ball, $\lambda^{*}=\frac{\lambda_{1}}{4}$ ). The main reason for this difference is that the function defined in (1.4) does not decay fast enough if $N=3$. Similarly, the main reason that we are not able to prove Theorem 1.1 for $N=3$ is that the function defined in (1.7) does not decay fast enough.

In the Lazer and McKenna conjecture, the parameter $s$ is large. Let us now consider the other extreme case: $s \rightarrow 0+$. Using the same argument as in [7], we can show that for $\lambda \in\left(\lambda^{*}, \lambda_{1}\right), \lambda^{*}=0$ if $N=4, \lambda^{*}>0$ if $N=3,(1.3)$ has a least energy solution if $s>0$ is small. We can obtain more in the case $N=3$.

Theorem 1.3. Suppose that $\Omega$ satisfies $(S 1)$ and $(S 2)$, and $N=3, \lambda<\lambda_{1}$. Then, the number of the solutions for (1.3) is unbounded as $s \rightarrow 0+$.

Note that the result in Theorem 1.3 is not trivial, because if $\lambda<\lambda^{*}$, we can not find even one solution by using the method in [7]. Moreover, we show that (1.3) has more and more solutions as $s \rightarrow 0+$ for all $\lambda<\lambda_{1}$ if $N=3$. 
The readers can refer to $[6,10,11,17]$ for results on the Lazer-McKenna conjecture for other type of nonlinearities.

In Theorems 1.1-1.3, we have assumed that $N \geq 3$. When $N=2, \mathrm{M}$. del Pino and Munoz [17] proved the Lazer-McKenna conjecture when the right hand nonlinearity is $e^{u}$ (which is still subcritical in $\mathbb{R}^{2}$ ). The authors believe that when $N=2$, results similar to Theorems 1.1-1.3 may be true if the right hand nonlinearity is of the critical type, i.e., $h(u) e^{u^{2}}$. When $N=1$, the critical exponent is $\frac{N+2}{N-2}=-3$. In this case, some form of Lazer-McKenna conjecture may be true if the right hand nonlinearity is $-u^{-3}$. We refer to [1] and [2] for discussions on critical nonlinearities in dimensions $N=1,2$.

Before we close this section, let us outline the proof of Theorems 1.1 and 1.2 and discuss the conditions imposed in these two theorems.

For any $\bar{x} \in \mathbb{R}^{N}, \mu>0$, denote

$$
U_{\mu, \bar{x}}(y)=(N(N-2))^{\frac{N-2}{4}} \frac{\mu^{(N-2) / 2}}{\left(1+\mu^{2}|y-\bar{x}|^{2}\right)^{(N-2) / 2}} .
$$

Then, $U_{\mu, \bar{x}}$ satisfies $-\Delta U_{\mu, \bar{x}}=U_{\mu, \bar{x}}^{2^{*}-1}$. In this paper, we will use the following notation: $U=U_{1,0}$.

Let

$$
\varepsilon=\frac{s^{\frac{2}{N-2}}}{k^{2}}, \quad \mu=\frac{\Lambda}{\varepsilon}, \quad \Lambda \in\left[\delta, \delta^{-1}\right]
$$

and $k \geq k_{0}$, where $\delta>0$ is a small constant, and $k_{0}>0$ is a large constant, which is to be determined later.

Using the transformation $u(y) \mapsto \varepsilon^{-\frac{N-2}{2}} u\left(\frac{y}{\varepsilon}\right)$, we find that (1.3) becomes

$$
\begin{cases}-\Delta u=\left(u-s \varepsilon^{\frac{N-2}{2}} \varphi_{1}(\varepsilon y)\right)_{+}^{2^{*}-1}+\lambda \varepsilon^{2} u, & \text { in } \Omega_{\varepsilon}, \\ u=0, & \text { on } \partial \Omega_{\varepsilon},\end{cases}
$$

where $\Omega_{\varepsilon}=\{y: \varepsilon y \in \Omega\}$. Let

$$
\Phi_{\varepsilon}(y)=\varepsilon^{\frac{N-2}{2}} \varphi_{1}(\varepsilon y) .
$$

For $\xi \in \Omega_{\varepsilon}$, we define $W_{\Lambda, \xi}$ as the unique solution of

$$
\begin{cases}-\Delta W-\lambda \varepsilon^{2} W=U_{\Lambda, \xi}^{2^{*}-1} & \text { in } \Omega_{\varepsilon} \\ W=0 & \text { on } \partial \Omega_{\varepsilon}\end{cases}
$$

Let $y=\left(y^{\prime}, y^{\prime \prime}\right) \in \mathbb{R}^{N}$, where $y^{\prime}=\left(y_{1}, y_{2}\right)$, and $y^{\prime \prime}=\left(y_{3}, \cdots, y_{N}\right)$. Define

$$
\begin{array}{r}
H_{S}=\left\{u: u \in H^{1}\left(\Omega_{\varepsilon}\right), u \text { is even in } y_{h}, h=3, \cdots, N, u\left(r \cos \theta, r \sin \theta, y^{\prime \prime}\right)\right. \\
\left.=u\left(r \cos \left(\theta+\frac{2 \pi j}{k}\right), r \sin \left(\theta+\frac{2 \pi j}{k}\right), y^{\prime \prime}\right), j=1, \ldots, k-1\right\},
\end{array}
$$


and

$$
\mathbf{x}_{j}=\left(\frac{r}{\varepsilon} \cos \frac{2(j-1) \pi}{k}, \frac{r}{\varepsilon} \sin \frac{2(j-1) \pi}{k}, 0\right), \quad j=1, \cdots, k,
$$

where 0 is the zero vector in $\mathbb{R}^{N-2}$.

Let

$$
W_{r, \Lambda}(y)=\sum_{j=1}^{k} W_{\Lambda, \mathbf{x}_{j}} .
$$

We are going to construct a solution for (1.3), which is close to $W_{r, \Lambda}$ for some suitable $\Lambda$ and $r$ and large $k$.

Theorem 1.1 is a direct consequence of the following result:

Theorem 1.4. Under the same conditions as in Theorem 1.1, there is an integer $k_{0}>0$, such that for any integer $k \geq k_{0}$, (1.5) has a solution $u_{k}$ of the form

$$
u_{k}=W_{r_{k}, \Lambda_{k}}(y)+\omega_{k},
$$

where $\omega_{k} \in H_{s}$, and as $k \rightarrow+\infty, r_{k} \rightarrow r_{0}>0, \Lambda_{k} \rightarrow \Lambda_{0}>0,\left\|\omega_{k}\right\|_{L^{\infty}} \rightarrow 0$.

On the other hand, if $N \geq 7$ and $\lambda<0$, we have the following weaker result:

Theorem 1.5. Suppose that $N \geq 7$ and $\lambda<\lambda_{1}$. Then there is a large constant $s_{0}>0$, such that for any $s>s_{0}$, and integer $k$ satisfying $s^{\frac{(2-2 \theta)(N-4)}{(N-6)(N-2)}} \leq k \leq$ $s^{\frac{(2-\theta)(N-4)}{(N-6)(N-2)}}$, where $\theta>0$ is a fixed small constant, (1.5) has a solution $u_{k, s}$ of the form

$$
u_{k, s}=W_{r_{k}, \Lambda_{k}}(y)+\omega_{k, s},
$$

where $\omega_{k, s} \in H_{s}$, and as $s \rightarrow+\infty, r_{k} \rightarrow r_{0}>0, \Lambda_{k} \rightarrow \Lambda_{0}>0,\left\|\omega_{k, s}\right\|_{L^{\infty}} \rightarrow 0$.

Since $s^{\frac{(2-\theta)(N-4)}{(N-6)(N-2)}}-s^{\frac{(2-2 \theta)(N-4)}{(N-6)(N-2)}} \rightarrow+\infty$ as $s \rightarrow+\infty$, Theorem 1.2 is a direct consequence of Theorem 1.5. Let us point out that in the case $N \geq 7$ and $\lambda \in$ $\left(0, \lambda_{1}\right)$, the solutions in Theorem 1.5 are different from those constructed in [27,36], where the energy of the solutions remains bounded as $s \rightarrow+\infty$.

It is easy to see that Theorem 1.3 is a direct consequence of the following result:

Theorem 1.6. Suppose that $N=3$ and $\lambda<\lambda_{1}$. Then there is a small constant $s_{1}>0$ and a large constant $k_{0}>0$ (independent of $s$ ), such that for any $s \in\left(0, s_{1}\right)$, and integer $k$ satisfying

$$
k_{0} \leq k \leq C s^{-\frac{2 \tau}{1-2 \tau}}
$$

for some $\tau \in\left(0, \frac{4}{11}\right)$, then (1.5) has a solution $u_{k, s}$ of the form

$$
u_{k, s}=W_{r_{k}, \Lambda_{k}}(y)+\omega_{k, s},
$$

where $\omega_{k, s} \in H_{s}$, and as $s \rightarrow 0, r_{k} \rightarrow r_{0}>0, \Lambda_{k} \rightarrow \Lambda_{0}>0,\left\|\omega_{k, s}\right\|_{L^{\infty}} \rightarrow 0$. 
Let make a few remarks on the conditions imposed on Theorems 1.1 and 1.2. It is easy to see that the first eigenfunction $\varphi_{1} \in H_{s}$. In this paper, we denote

$$
\bar{\varphi}(r)=\varphi_{1}(r, 0) .
$$

The functional corresponding to (1.5) is

$$
I(u)=\frac{1}{2} \int_{\Omega_{\varepsilon}}\left(|D u|^{2}-\lambda \varepsilon^{2} u^{2}\right)-\frac{1}{2^{*}} \int_{\Omega_{\varepsilon}}\left(u-s \Phi_{\varepsilon}\right)_{+}^{2^{*}}, \quad u \in H_{s} .
$$

Let $\Gamma$ be a connected component of the set $\Omega \cap\left\{y_{3}=\cdots=y_{N}=0\right\}$. Then, by $(S 1)$, there are $r_{2}>r_{1} \geq 0$, such that

$$
\bar{\Gamma}=\left\{y: r_{1} \leq \sqrt{y_{1}^{2}+y_{2}^{2}} \leq r_{2}, y_{3}=\cdots=y_{N}=0\right\} .
$$

If $N=4,5$, then $\frac{N-2}{2}<2$. We obtain from Proposition A.3,

$$
I\left(W_{r, \Lambda}\right)=k\left(A_{0}+\frac{A_{2} s \bar{\varphi}(r) \varepsilon^{\frac{N-2}{2}}}{\Lambda^{\frac{N-2}{2}}}-\frac{A_{3} \varepsilon^{N-2} k^{N-2}}{r^{N-2} \Lambda^{N-2}}+O\left(\varepsilon^{\frac{(N-2)(1+\sigma)}{2}}\right)\right) .
$$

It is easy to see that the function

$$
r^{\frac{N-2}{2}} \bar{\varphi}(r), \quad r \in\left[r_{1}, r_{2}\right]
$$

has a maximum point $r_{0}$, satisfying $r_{0} \in\left(r_{1}, r_{2}\right)$, since $r_{i}^{\frac{N-2}{2}} \bar{\varphi}\left(r_{i}\right)=0, i=1,2$. As a result,

$$
\frac{A_{2} s \bar{\varphi}(r)}{\Lambda^{\frac{N-2}{2}}}-\frac{A_{3}}{r^{N-2} \Lambda^{N-2}}, \quad(r, \Lambda) \in\left(r_{1}, r_{2}\right) \times\left(\delta, \delta^{-1}\right),
$$

has a maximum point $\left(r_{0}, \Lambda_{0}\right)$, where

$$
\Lambda_{0}=\left(\frac{2 A_{3}}{A_{2} s r_{0}^{N-2} \bar{\varphi}\left(r_{0}\right)}\right)^{\frac{2}{N-2}}
$$

for any fixed $s>0$. Thus, $I\left(W_{r, \Lambda}\right)$ has a maximum point in $\left(r_{1}, r_{2}\right) \times\left(\delta, \delta^{-1}\right)$, if $k>0$ is large.

If $N=6$, then $\frac{N-2}{2}=2$. Thus, we find from Proposition A.3,

$$
I\left(W_{r, \Lambda}\right)=k\left(A_{0}+\left(-\lambda A_{1}+A_{2} s \bar{\varphi}(r)\right) \frac{\varepsilon^{2}}{\Lambda^{2}}-\frac{A_{3} \varepsilon^{4} k^{4}}{r^{4} \Lambda^{4}}+O\left(\varepsilon^{2+\sigma}\right)\right) .
$$

Let

$$
g(r)=r^{2}\left(A_{2} s \bar{\varphi}(r)-A_{1} \lambda\right), \quad r \in\left[r_{1}, r_{2}\right]
$$


It is easy to see that we can always choose a constant $s_{0}>0$, such that if $s>|\lambda| s_{0}$, then $g(r)$ has a maximum point $r_{0}$, satisfying $g\left(r_{0}\right)>0, r_{0} \in\left(r_{1}, r_{2}\right)$. As a result,

$$
\frac{-\lambda A_{1}+A_{2} s \bar{\varphi}(r)}{\Lambda^{2}}-\frac{A_{3}}{r^{4} \Lambda^{4}}, \quad(r, \Lambda) \in\left(r_{1}, r_{2}\right) \times\left(\delta, \delta^{-1}\right),
$$

has maximum point $\left(r_{0}, \Lambda_{0}\right)$, where

$$
\Lambda_{0}=\left(\frac{2 A_{3}}{\left(-\lambda A_{1}+A_{2} s \bar{\varphi}\left(r_{0}\right)\right) r_{0}^{4}}\right)^{\frac{1}{2}}
$$

for any fixed $s>0$. Thus, $I\left(W_{r, \lambda}\right)$ has a maximum point in $\left(r_{1}, r_{2}\right) \times\left(\delta, \delta^{-1}\right)$, if $k>0$ is large.

If $N \geq 7$ and $\lambda=0$, then Proposition A.3 gives

$$
I\left(W_{r, \Lambda}\right)=k\left(A_{0}+\frac{A_{2} s \bar{\varphi}(r) \varepsilon^{\frac{N-2}{2}}}{\Lambda^{\frac{N-2}{2}}}-\frac{A_{3} \varepsilon^{N-2} k^{N-2}}{r^{N-2} \Lambda^{N-2}}+O\left(\varepsilon^{\frac{(N-2)(1+\sigma)}{2}}\right)\right),
$$

So, we are in the same situation as the case $N=4,5$.

On the other hand, if $N \geq 7$, then $\frac{N-2}{2}>2$. Thus $\varepsilon^{\frac{N-2}{2}}$ is a higher order term of $\varepsilon^{2}$. Thus if $\lambda \neq 0$, then for each fixed $s>0$, we have

$$
I\left(W_{r, \Lambda}\right)=k\left(A_{0}-\frac{\lambda A_{1} \varepsilon^{2}}{\Lambda^{2}}-\frac{A_{3} \varepsilon^{N-2} k^{N-2}}{r^{N-2} \Lambda^{N-2}}+O\left(\varepsilon^{2+\sigma}\right)\right),
$$

But

$$
-\frac{\lambda A_{1}}{\Lambda^{2}}-\frac{A_{3}}{r^{N-2} \Lambda^{N-2}}, \quad(r, \Lambda) \in\left(r_{1}, r_{2}\right) \times\left(\delta, \delta^{-1}\right),
$$

does not have a critical point even if $\lambda<0$. So, we don't know whether $I\left(W_{r, \Lambda}\right)$ has a critical point. Thus, to obtain a solution for (1.3), we need to let $s$ change so that

$$
\varepsilon^{2} \ll s \varepsilon^{\frac{N-2}{2}}, \quad \varepsilon \ll 1 .
$$

If (1.15) holds, then

$$
I\left(W_{r, \Lambda}\right)=k\left(A_{0}+\frac{A_{2} \bar{\varphi}(r) s \varepsilon^{\frac{N-2}{2}}}{\Lambda^{\frac{N-2}{2}}}-\frac{A_{3} \varepsilon^{N-2} k^{N-2}}{r^{N-2} \Lambda^{N-2}}+O\left(\left(s \varepsilon^{\frac{N-2}{2}}\right)^{1+\sigma}\right)\right) .
$$

So, we are in a similar situation as $\lambda=0$. Note the (1.15) implies

$$
k \ll s^{\frac{2(N-4)}{(N-2)(N-6)}}, \quad k \gg s^{\frac{1}{N-2}},
$$

which gives an upper bound for $k$. Therefore, in this case, we are not able to obtain the existence of infinitely many solutions even if $s>0$ is large. 
In the case $N=3$, for fixed $s>0$, some estimates which are valid for $N \geq 4$ may not be true due to the slow decay of the function $W_{r, \Lambda}$. Under the condition $s \leq C k^{-\frac{1}{2 \tau}+1}$ for some $\tau \in\left(0, \frac{4}{11}\right)$, we can recover all these estimates. But the condition $s \leq C k^{-\frac{1}{2 \tau}+1}$ imposes an upper bound (1.8) for the number of bubbles $k$.

The energy of the solutions obtained in Theorems 1.4 and 1.5 is very large because $k$ must be large. This result is in consistence of the result in [21].

Finally, let us point out that the eigenvalue $\varphi_{1}$ is not essential in this paper. We can replace $\varphi_{1}$ by any function $\varphi$, satisfying $\varphi>0$ in $\Omega, \varphi=0$ on $\partial \Omega$ and $\varphi \in H_{s}$.

We will use the reduction argument as in $[4,5,14-16,29,30]$ and [38] to prove the main results of this paper. Unlike those papers, where a parameter always appears in some form, in Theorem 1.4, $s$ is a fixed positive constant. To prove Theorem 1.4, the number of the bubbles $k$ is used as a parameter to carry out the reduction. Similar idea has been used in [35,37].

\section{The reduction}

In this section, we will reduce the problem of finding a $k$-peak solution for (1.3) to a finite dimension problem.

Let

$$
\|u\|_{*}=\sup _{y}\left(\sum_{j=1}^{k} \frac{1}{\left(1+\left|y-\mathbf{x}_{j}\right|\right)^{\frac{N-2}{2}+\tau}}\right)^{-1}|u(y)|,
$$

and

$$
\|f\|_{* *}=\sup _{y}\left(\sum_{j=1}^{k} \frac{1}{\left(1+\left|y-\mathbf{x}_{j}\right|\right)^{\frac{N+2}{2}+\tau}}\right)^{-1}|f(y)|,
$$

where $\tau \in(0,1)$ is a constant, such that

$$
\sum_{j=2}^{k} \frac{1}{\left|\mathbf{x}_{j}-\mathbf{x}_{1}\right|^{\tau}} \leq C
$$

Recall that $\varepsilon=\frac{\frac{2}{N-2}}{k^{2}}$, and

$$
\sum_{j=2}^{k} \frac{1}{\left|\mathbf{x}_{j}-\mathbf{x}_{1}\right|^{\tau}} \leq C \varepsilon^{\tau} k^{\tau} \sum_{j=2}^{k} \frac{1}{j^{\tau}} \leq C \varepsilon^{\tau} k .
$$

In order to achieve (2.3), we need to choose $\tau$ according to whether $s>0$ is fixed or not. We choose $\tau$ as follows:

$$
\tau= \begin{cases}\frac{1}{2}, & \text { in Theorems 1.4 and 1.5; } \\ \text { the number in (1.8), } & \text { in Theorem 1.6. }\end{cases}
$$


Let

$$
Y_{i, 1}=\frac{\partial W_{\Lambda, \mathbf{x}_{i}}}{\partial \Lambda}, \quad Z_{i, 1}=-\Delta Y_{i, 1}-\lambda \varepsilon^{2} Y_{i, 1}=\left(2^{*}-1\right) U_{\Lambda, \mathbf{x}_{i}}^{2^{*}-2} \frac{\partial U_{\Lambda, \mathbf{x}_{i}}}{\partial \Lambda},
$$

and

$$
Y_{i, 2}=\frac{\partial W_{\Lambda, \mathbf{x}_{i}}}{\partial r}, \quad Z_{i, 2}=-\Delta Y_{i, 2}-\lambda \varepsilon^{2} Y_{i, 2}=\left(2^{*}-1\right) U_{\Lambda, \mathbf{x}_{i}}^{2^{*}-2} \frac{\partial U_{\Lambda, \mathbf{x}_{i}}}{\partial r}
$$

We consider

$$
\left\{\begin{array}{l}
-\Delta \phi_{k}-\lambda \varepsilon^{2} \phi_{k}-\left(2^{*}-1\right)\left(W_{r, \Lambda}-s \Phi_{\varepsilon}\right)_{+}^{2^{*}-2} \phi_{k}=h+\sum_{j=1}^{2} \sum_{i=1}^{k} c_{j} Z_{i, j}, \text { in } \Omega_{\varepsilon}, \\
\phi_{k} \in H_{s}, \\
\left\langle\sum_{i=1}^{k} Z_{i, j}, \phi_{k}\right\rangle=0, \quad j=1,2,
\end{array}\right.
$$

for some number $c_{j}$, where $\langle u, v\rangle=\int_{\Omega_{\varepsilon}} u v$.

We need the following result, whose proof is standard.

Lemma 2.1. Let $f$ satisfy $\|f\|_{* *}<\infty$ and let $u$ be the solution of

$$
-\Delta u-\lambda \varepsilon^{2} u=f \quad \text { in } \quad \Omega_{\varepsilon}, \quad u=0 \quad \text { on } \partial \Omega_{\varepsilon},
$$

where $\lambda<\lambda_{1}$. Then we have

$$
|u(y)| \leq C \int_{\Omega_{\varepsilon}} \frac{|f(z)|}{|z-y|^{N-2}} d z .
$$

Next, we need the following lemma to carry out the reduction.

Lemma 2.2. Assume that $\phi_{k}$ solves (2.5) for $h=h_{k}$. If $\left\|h_{k}\right\|_{* *}$ goes to zero as $k$ goes to infinity, so does $\left\|\phi_{k}\right\|_{*}$.

Proof. We argue by contradiction. Suppose that there are $k \rightarrow+\infty, h=h_{k}$, $\Lambda_{k} \in\left[\delta, \delta^{-1}\right]$, and $\phi_{k}$ solving (2.5) for $h=h_{k}, \Lambda=\Lambda_{k}$, with $\left\|h_{k}\right\|_{* *} \rightarrow 0$, and $\left\|\phi_{k}\right\|_{*} \geq c^{\prime}>0$. We may assume that $\left\|\phi_{k}\right\|_{*}=1$. For simplicity, we drop the subscript $k$.

By Lemma 2.1,

$$
\begin{aligned}
|\phi(y)| \leq & C \int_{\Omega_{\varepsilon}} \frac{1}{|z-y|^{N-2}} W_{r, \Lambda}^{2^{*}-2}|\phi(z)| d z \\
& +C \int_{\Omega_{\varepsilon}} \frac{1}{|z-y|^{N-2}}\left(|h(z)|+\left|\sum_{j=1}^{2} \sum_{i=1}^{k} c_{j} Z_{i, j}(z)\right|\right) d z
\end{aligned}
$$


Using Lemma B.4 and B.5, there is a strictly positive number $\theta$ such that

$$
\left|\int_{\Omega_{\varepsilon}} \frac{1}{|z-y|^{N-2}} W_{r, \Lambda}^{2^{*}-2} \phi(z) d z\right| \leq C\|\phi\|_{*} \sum_{j=1}^{k} \frac{1}{\left(1+\left|y-\mathbf{x}_{j}\right|\right)^{\frac{N-2}{2}+\tau+\theta}} .
$$

It follows from Lemma B.3 that

$$
\begin{aligned}
\left|\int_{\Omega_{\varepsilon}} \frac{1}{|z-y|^{N-2}} h(z) d z\right| & \leq C\|h\|_{* *} \int_{\mathbb{R}^{N}} \frac{1}{|z-y|^{N-2}} \sum_{j=1}^{k} \frac{1}{\left(1+\left|z-\mathbf{x}_{j}\right|\right)^{\frac{N+2}{2}+\tau}} d z \\
& \leq C\|h\|_{* *} \sum_{j=1}^{k} \frac{1}{\left(1+\left|y-\mathbf{x}_{j}\right|\right)^{\frac{N-2}{2}+\tau}}
\end{aligned}
$$

and

$$
\begin{aligned}
\left|\int_{\Omega_{\varepsilon}} \frac{1}{|z-y|^{N-2}} \sum_{i=1}^{k} Z_{i, j}(z) d z\right| & \leq C \sum_{i=1}^{k} \int_{\mathbb{R}^{N}} \frac{1}{|z-y|^{N-2}} \frac{1}{\left(1+\left|z-\mathbf{x}_{i}\right|\right)^{N+2}} d z \\
& \leq C \sum_{i=1}^{k} \frac{1}{\left(1+\left|y-\mathbf{x}_{i}\right|\right)^{\frac{N-2}{2}+\tau}}
\end{aligned}
$$

Next, we estimate $c_{j}$. Multiplying (2.5) by $Y_{1, l}$ and integrating, we see that $c_{j}$ satisfies

$$
\left\langle\sum_{j=1}^{2} \sum_{i=1}^{k} Z_{i, j}, Y_{1, l}\right\rangle c_{j}=\left\langle-\Delta \phi-\lambda \varepsilon^{2} \phi-\left(2^{*}-1\right) W_{r, \Lambda}^{2^{*}-2} \phi, Y_{1, l}\right\rangle-\left\langle h, Y_{1, l}\right\rangle .
$$

It follows from Lemma B.2 that

$$
\left|\left\langle h, Y_{1, l}\right\rangle\right| \leq C\|h\|_{* *} \int_{\mathbb{R}^{N}} \frac{1}{\left(1+\left|z-\mathbf{x}_{1}\right|\right)^{N-2-\beta}} \sum_{j=1}^{k} \frac{1}{\left(1+\left|z-\mathbf{x}_{j}\right|\right)^{\frac{N+2}{2}+\tau}} d z \leq C\|h\|_{* *},
$$

since $\beta>0$ can be chosen as small as desired. 
On the other hand,

$$
\begin{aligned}
& \left\langle-\Delta \phi-\lambda \varepsilon^{2} \phi-\left(2^{*}-1\right) W_{r, \Lambda}^{2^{*}-2} \phi, Y_{1, l}\right\rangle \\
& =\left\langle-\Delta Y_{1, l}-\lambda \varepsilon^{2} Y_{1, l}-\left(2^{*}-1\right) W_{r, \Lambda}^{2^{*}-2} Y_{1, l}, \phi\right\rangle \\
& =\left(2^{*}-1\right)\left\langle U_{\Lambda, \mathbf{x}_{1}}^{2^{*}-2} \partial_{l} U_{\Lambda, \mathbf{x}_{1}}-W_{r, \Lambda}^{2^{*}-2} Y_{1, l}, \phi\right\rangle,
\end{aligned}
$$

where $\partial_{l}=\partial_{\Lambda}$ if $l=1, \partial_{l}=\partial_{r}$ if $l=2$.

By Lemmas B.1,

$$
|\phi(y)| \leq C\|\phi\|_{*} .
$$

We consider the cases $N \geq 6$ first. Note that $\frac{4}{N-2} \leq 1$ for $N \geq 6$. Using Lemmas A.1 and B.2, noting that

$$
\left|W_{r, \Lambda}^{2^{*}-2}-W_{\Lambda, \mathbf{x}_{1}}^{2^{*}-2}\right| \leq \sum_{j=2}^{k} W_{\Lambda, \mathbf{x}_{j}}^{2^{*}-2}
$$

and

$$
\varepsilon \leq \frac{C}{1+\left|z-\mathbf{x}_{1}\right|}
$$

we obtain

$$
\begin{aligned}
& \quad\left|\left\langle U_{\Lambda, \mathbf{x}_{1}}^{2^{*}-2} \partial_{l} U_{\Lambda, x_{j}}-W_{r, \Lambda}^{2^{*}-2} Y_{1, l}, \phi\right\rangle\right| \\
& \leq C\|\phi\|_{*} \int_{\Omega_{\varepsilon}} \frac{1}{\left(1+\left|z-\mathbf{x}_{1}\right|\right)^{N-2-\beta}} \sum_{i=2}^{k} \frac{1}{\left(1+\left|z-\mathbf{x}_{i}\right|\right)^{4-\beta}} d z \\
& \quad+C\|\phi\|_{*} \int_{\Omega_{\varepsilon}} U_{\Lambda, \mathbf{x}_{1}}^{2^{*}-2}\left(\varepsilon^{N-2}+\frac{|\lambda| \varepsilon^{2}}{\left(1+\left|y-\mathbf{x}_{j}\right|\right)^{N-4-\beta}}\right) \sum_{j=1}^{k} \frac{1}{\left(1+\left|z-\mathbf{x}_{j}\right|\right)^{\frac{N-2}{2}+\tau}} \\
& \quad+C\|\phi\|_{*} \int_{\Omega_{\varepsilon}} U_{\Lambda, \mathbf{x}_{1}}\left(\varepsilon^{N-2}+\frac{|\lambda| \varepsilon^{2}}{\left(1+\left|y-\mathbf{x}_{j}\right|\right)^{N-4-\beta}}\right)^{2^{*}-2} \sum_{j=1}^{k} \frac{1}{\left(1+\left|z-\mathbf{x}_{j}\right|\right)^{\frac{N-2}{2}+\tau}} \\
& \leq C\|\phi\|_{*} \sum_{j=2}^{k} \frac{1}{\left|\mathbf{x}_{1}-\mathbf{x}_{j}\right|^{1+\sigma}}+o(1)\|\phi\|_{*}=o(1)\|\phi\|_{*} .
\end{aligned}
$$


For $N=3,4,5$, we have $\frac{4}{N-2}>1$. By Lemmas B.1, B.2,

$$
\begin{aligned}
& \quad\left|\left\langle U_{\Lambda, \mathbf{x}_{1}}^{2^{*}-2} \partial_{l} U_{\Lambda, \mathbf{x}_{j}}-W_{r, \Lambda}^{2^{*}-2} Y_{1, l}, \phi\right\rangle\right| \\
& \leq C \int_{\Omega_{\varepsilon}} W_{\Lambda, \mathbf{x}_{1}}^{2^{*}-3} \sum_{j=2}^{k} W_{\Lambda, \mathbf{x}_{j}}\left|Y_{1, l} \phi\right|+C \int_{\Omega_{\varepsilon}}\left(\sum_{j=2}^{k} W_{\Lambda, \mathbf{x}_{j}}\right)^{\frac{4}{N-2}}\left|Y_{1} \phi\right| \\
& \quad+C\|\phi\|_{*} \int_{\Omega_{\varepsilon}} U_{\Lambda, \mathbf{x}_{1}}^{2^{*}-2}\left(\varepsilon^{N-2}+\frac{|\lambda| \varepsilon^{2}}{\left(1+\left|y-\mathbf{x}_{j}\right|\right)^{N-4-\beta}}\right) \sum_{j=1}^{k} \frac{1}{\left(1+\left|z-\mathbf{x}_{j}\right|\right)^{\frac{N-2}{2}+\tau}} \\
& \quad+C\|\phi\|_{*} \int_{\Omega_{\varepsilon}} U_{\Lambda, \mathbf{x}_{1}}\left(\varepsilon^{N-2}+\frac{|\lambda| \varepsilon^{2}}{\left(1+\left|y-\mathbf{x}_{j}\right|\right)^{N-4-\beta}}\right)^{2^{*}-2} \sum_{j=1}^{k} \frac{1}{\left(1+\left|z-\mathbf{x}_{j}\right|\right)^{\frac{N-2}{2}+\tau}} \\
& \leq C\|\phi\|_{*} \int_{\Omega_{\varepsilon}} \frac{1}{\left(1+\left|z-\mathbf{x}_{1}\right|\right)^{4-\beta}} \sum_{j=2}^{k} \frac{1}{\left(1+\left|z-\mathbf{x}_{j}\right|\right)^{N-2-\beta}} \\
& \quad+C \int_{\Omega_{\varepsilon}}\left(\sum_{j=2}^{k} U_{\Lambda, \mathbf{x}_{j}}^{1-\beta}\right)^{\frac{4}{N-2}}\left|Y_{1, l} \phi\right|+o(1)\|\phi\|_{*} \\
& \leq C\|\phi\|_{*} \int_{\Omega_{\varepsilon}} \frac{1}{\left(1+\left|z-\mathbf{x}_{1}\right|\right)^{N-2-\beta}}\left(\sum_{j=2}^{k} U_{\Lambda, \mathbf{x}_{j}}^{1-\beta}\right)^{\frac{4}{N-2}} \sum_{i=1}^{k} \frac{1}{\left(1+\left|y-\mathbf{x}_{i}\right|\right)^{\frac{N-2}{2}+\tau}} \\
& \quad+o(1)\|\phi\|_{*} .
\end{aligned}
$$

Let

$$
\Omega_{j}=\left\{y=\left(y^{\prime}, y^{\prime \prime}\right) \in \Omega_{\varepsilon}:\left\langle\frac{y^{\prime}}{\left|y^{\prime}\right|}, \frac{\mathbf{x}_{j}}{\left|\mathbf{x}_{j}\right|}\right\rangle \geq \cos \frac{\pi}{k}\right\} .
$$

If $y \in \Omega_{1}$, then

$$
\begin{aligned}
\sum_{j=2}^{k} U_{\Lambda, \mathbf{x}_{j}}^{1-\beta} & \leq \frac{1}{\left(1+\left|y-\mathbf{x}_{1}\right|\right)^{N-2-\tau-(N-2) \beta-\theta}} \sum_{j=2}^{k} \frac{1}{\left|\mathbf{x}_{j}-\mathbf{x}_{1}\right|^{\tau+\theta}} \\
& =o(1) \frac{1}{\left(1+\left|y-\mathbf{x}_{1}\right|\right)^{N-2-\tau-(N-2) \beta-\theta}}
\end{aligned}
$$

and

$$
\sum_{i=1}^{k} \frac{1}{\left(1+\left|y-\mathbf{x}_{i}\right|\right)^{\frac{N-2}{2}+\tau}} \leq \frac{C}{\left(1+\left|y-\mathbf{x}_{1}\right|\right)^{\frac{N-2}{2}}} .
$$


So, we obtain

$$
\begin{aligned}
& \int_{\Omega_{1}} \frac{1}{\left(1+\left|z-\mathbf{x}_{1}\right|\right)^{N-2-\beta}}\left(\sum_{j=2}^{k} U_{\Lambda, \mathbf{x}_{j}}^{1-\beta}\right)^{\frac{4}{N-2}} \sum_{i=1}^{k} \frac{1}{\left(1+\left|y-\mathbf{x}_{i}\right|\right)^{\frac{N-2}{2}+\tau}} \\
& =o(1) \int_{\Omega_{1}} \frac{1}{\left(1+\left|z-\mathbf{x}_{1}\right|\right)^{N+\frac{N+2}{2}-\frac{4(\tau+\theta)}{N-2}-4 \beta}}=o(1),
\end{aligned}
$$

since $\frac{N+2}{2}-\frac{4(\tau+\theta)}{N-2}-4 \beta>0$, if $\beta>0$ and $\theta>0$ are small.

If $y \in \Omega_{l}, l \geq 2$, then

$$
\sum_{j=2}^{k} U_{\Lambda, \mathbf{x}_{j}}^{1-\beta} \leq \frac{C}{\left(1+\left|y-\mathbf{x}_{l}\right|\right)^{N-2-\tau-(N-2) \beta}},
$$

and

$$
\sum_{i=1}^{k} \frac{1}{\left(1+\left|y-\mathbf{x}_{i}\right|\right)^{\frac{N-2}{2}+\tau}} \leq \frac{C}{\left(1+\left|y-\mathbf{x}_{l}\right|\right)^{\frac{N-2}{2}}} .
$$

As a result,

$$
\begin{aligned}
& \int_{\Omega_{l}} \frac{1}{\left(1+\left|z-\mathbf{x}_{1}\right|\right)^{N-2}}\left(\sum_{j=2}^{k} U_{\Lambda, \mathbf{x}_{j}}^{1-\beta}\right)^{\frac{4}{N-2}} \sum_{i=1}^{k} \frac{1}{\left(1+\left|y-\mathbf{x}_{i}\right|\right)^{\frac{N-2}{2}+\tau}} \\
& \leq C \int_{\Omega_{l}} \frac{1}{\left(1+\left|z-\mathbf{x}_{1}\right|\right)^{N-2}} \frac{1}{\left(1+\left|y-\mathbf{x}_{l}\right|\right)^{4-4 \beta-\frac{4 \tau}{N-2}+\frac{N-2}{2}}} \\
& \leq \frac{C}{\left|\mathbf{x}_{l}-\mathbf{x}_{1}\right|^{\frac{N+2}{2}-\frac{4 \tau}{N-2}-\theta-4 \beta}}
\end{aligned}
$$

where $\theta>0$ is a fixed small constant.

Since $\tau=\frac{1}{2}$ for $N \geq 4$, and $\tau<\frac{1}{2}$ for $N=3$, we find that for $\theta>0$ and $\beta>0$ small, $\frac{N+2}{2}-\frac{4 \tau}{N-2}-\theta-4 \beta>\tau$. Thus

$$
\begin{aligned}
& \int_{\Omega_{\varepsilon}} \frac{1}{\left(1+\left|z-\mathbf{x}_{1}\right|\right)^{N-2}}\left(\sum_{j=2}^{k} U_{\Lambda, \mathbf{x}_{j}}^{1-\beta}\right)^{\frac{4}{N-2}} \sum_{i=1}^{k} \frac{1}{\left(1+\left|y-\mathbf{x}_{i}\right|\right)^{\frac{N-2}{2}+\tau}} \\
& \leq o(1)+C \sum_{l=2}^{k} \frac{1}{\left|\mathbf{x}_{l}-\mathbf{x}_{1}\right|^{\frac{N+2}{2}-\frac{4 \tau}{N-2}-\theta}}=o(1) .
\end{aligned}
$$

So, we have proved

$$
\left|\left\langle U_{\Lambda, \mathbf{x}_{1}}^{2^{*}-2} \partial_{l} U_{\Lambda, \mathbf{x}_{j}}-W_{r, \Lambda}^{2^{*}-2} Y_{1}, \phi\right\rangle\right|=o(1)\|\phi\|_{*} .
$$


But there is a constant $\bar{c}>0$,

$$
\left\langle\sum_{j=1}^{2} \sum_{i=1}^{k} Z_{i, j}, Y_{1, l}\right\rangle=\bar{c} \delta_{l j}+o(1) .
$$

Thus we obtain that

$$
c_{l}=o\left(\|\phi\|_{*}\right)+O\left(\|h\|_{* *}\right) .
$$

So,

$$
\|\phi\|_{*} \leq\left(o(1)+\left\|h_{k}\right\|_{* *}+\frac{\sum_{j=1}^{k} \frac{1}{\left(1+\left|y-\mathbf{x}_{j}\right|\right)^{\frac{N-2}{2}+\tau+\theta}}}{\sum_{j=1}^{k} \frac{1}{\left(1+\left|y-\mathbf{x}_{j}\right|\right)^{\frac{N-2}{2}+\tau}}}\right) .
$$

Since $\|\phi\|_{*}=1$, we obtain from (2.14) that there is $R>0$, such that

$$
\|\phi(y)\|_{B_{R}\left(\mathbf{x}_{i}\right) \geq c_{0}>0}
$$

for some $i$. But $\bar{\phi}(y)=\phi\left(y-\mathbf{x}_{i}\right)$ converges uniformly in any compact set of $\mathbb{R}_{+}^{N}$ to a solution $u$ of

$$
\Delta u+\left(2^{*}-1\right) U_{\Lambda, 0}^{2^{*}-2} u=0
$$

for some $\Lambda \in\left[\delta, \delta^{-1}\right]$, and $u$ is perpendicular to the kernel of (2.16). So, $u=0$. This is a contradiction to (2.15).

From Lemma 2.2, using the same argument as in the proof of [14, Proposition 4.1], we can prove the following result :

Proposition 2.3. There exists $k_{0}>0$ and a constant $C>0$, independent of $k$, such that for all $k \geq k_{0}$ and all $h \in L^{\infty}\left(\Omega_{\varepsilon}\right)$, problem (2.5) has a unique solution $\phi \equiv L_{k}(h)$. Besides,

$$
\left\|L_{k}(h)\right\|_{*} \leq C\|h\|_{* *}, \quad\left|c_{j}\right| \leq C\|h\|_{* *} .
$$

Moreover, the map $L_{k}(h)$ is $C^{1}$ with respect to $\Lambda$.

Now, we consider

$$
\left\{\begin{array}{l}
-\Delta\left(W_{r, \Lambda}+\phi\right)-\lambda \varepsilon^{2}\left(W_{r, \Lambda}+\phi\right)=\left(W_{r, \Lambda}+\phi-s \Phi_{\varepsilon}\right)_{+}^{2^{*}-1} \\
\quad+\sum_{j=1}^{2} \sum_{i=1}^{k} c_{j} Z_{i, j}, \quad \text { in } \Omega_{\varepsilon}, \\
\phi \in H_{s}, \\
\left\langle\sum_{i=1}^{k} Z_{i, j}, \phi\right\rangle=0, \quad j=1,2 .
\end{array}\right.
$$


We have:

Proposition 2.4. There is an integer $k_{0}>0$, such that for each $k \geq k_{0}, r_{1} \leq r \leq$ $r_{2}, \delta \leq \Lambda \leq \delta^{-1}$, where $\delta$ is a fixed small constant, (2.18) has a unique solution $\phi$, satisfying

$$
\|\phi\|_{*} \leq C\left(s \varepsilon^{\frac{N-2}{2}}\right)^{\frac{1}{2}+\sigma}+C|\lambda| \varepsilon^{1+\sigma},
$$

where $\sigma>0$ is a fixed small constant. Moreover, $\Lambda \rightarrow \phi(\Lambda)$ is $C^{1}$.

Rewrite (2.18) as

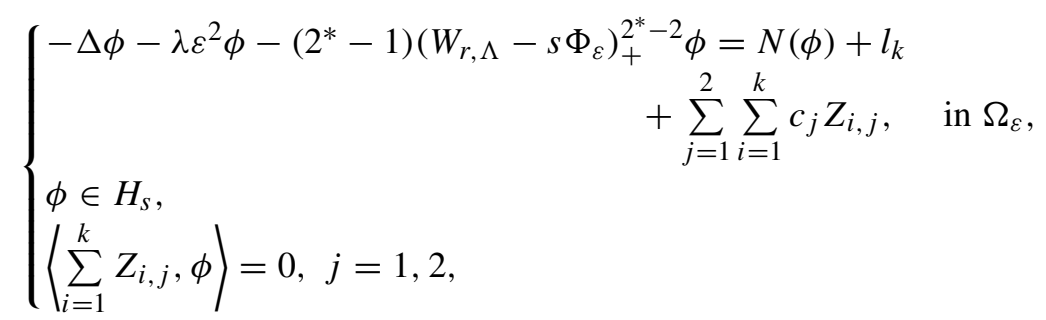

where

$\bar{N}(\phi)=\left(W_{r, \Lambda}-s \Phi_{\varepsilon}+\phi\right)_{+}^{2^{*}-1}-\left(W_{r, \Lambda}-s \Phi_{\varepsilon}\right)_{+}^{2^{*}-1}-\left(2^{*}-1\right)\left(W_{r, \Lambda}-s \Phi_{\varepsilon}\right)_{+}^{2^{*}-2} \phi$, and

$$
l_{k}=\left(W_{r, \Lambda}^{2^{*}-1}-\sum_{j=1}^{k} U_{\Lambda, x_{j}}^{2^{*}-1}\right)+\left(W_{r, \Lambda}-s \Phi_{\varepsilon}\right)_{+}^{2^{*}-1}-W_{r, \Lambda}^{2^{*}-1} .
$$

In order to use the contraction mapping theorem to prove that (2.19) is uniquely solvable in the set on which $\|\phi\|_{*}$ is small, we need to estimate $N(\phi)$ and $l_{k}$.

Lemma 2.5. We have

$$
\|\bar{N}(\phi)\|_{* *} \leq C\|\phi\|_{*}^{\min \left(2^{*}-1,2\right)}
$$

Proof. We have

$$
|\bar{N}(\phi)| \leq \begin{cases}C|\phi|^{2^{*}-1}, & N \geq 6 \\ C\left(W_{r, \Lambda}^{\frac{6-N}{N-2}} \phi^{2}+|\phi|^{2^{*}-1}\right), & N=3,4,5 .\end{cases}
$$

Firstly, we consider $N \geq 6$. We have

$$
\begin{aligned}
|\bar{N}(\phi)| & \leq C\|\phi\|_{*}^{2^{*}-1}\left(\sum_{j=1}^{k} \frac{1}{\left(1+\left|y-\mathbf{x}_{j}\right|\right)^{\frac{N-2}{2}+\tau}}\right)^{2^{*}-1} \\
& \leq C\|\phi\|_{*}^{2^{*}-1} \sum_{j=1}^{k} \frac{1}{\left(1+\left|y-\mathbf{x}_{j}\right|\right)^{\frac{N+2}{2}+\tau}}\left(\sum_{j=1}^{k} \frac{1}{\left(1+\left|y-\mathbf{x}_{j}\right|\right)^{\tau}}\right)^{\frac{4}{N-2}} \\
& \leq C\|\phi\|_{*}^{2^{*}-1} \sum_{j=1}^{k} \frac{1}{\left(1+\left|y-\mathbf{x}_{j}\right|\right)^{\frac{N+2}{2}+\tau}}
\end{aligned}
$$


where we use the inequality

$$
\sum_{j=1}^{k} a_{j} b_{j} \leq\left(\sum_{j=1}^{k} a_{j}^{p}\right)^{\frac{1}{p}}\left(\sum_{j=1}^{k} b_{j}^{q}\right)^{\frac{1}{q}}, \quad \frac{1}{p}+\frac{1}{q}=1, a_{j}, b_{j} \geq 0, j=1, \ldots, k
$$

and

$$
\sum_{j=1}^{k} \frac{1}{\left(1+\left|y-\mathbf{x}_{j}\right|\right)^{\tau}} \leq C+\sum_{j=2}^{k} \frac{C}{\left|\mathbf{x}_{1}-\mathbf{x}_{j}\right|^{\tau}} \leq C
$$

which follows from Lemma B.1.

For $N=3,4,5$, similarly to the case $N \geq 6$, we have

$$
\begin{aligned}
& |\bar{N}(\phi)| \\
\leq & C \phi \phi \|_{*}^{2}\left(\sum_{j=1}^{k} \frac{1}{\left(1+\left|y-\mathbf{x}_{j}\right|\right)^{N-2-\beta}}\right)^{\frac{6-N}{N-2}}\left(\sum_{j=1}^{k} \frac{1}{\left(1+\left|y-\mathbf{x}_{j}\right|\right)^{\frac{N-2}{2}+\tau}}\right)^{2} \\
& +C\|\phi\|_{*}^{2^{*}-1} \sum_{j=1}^{k} \frac{1}{\left(1+\left|y-\mathbf{x}_{j}\right|\right)^{\frac{N+2}{2}+\tau}} \\
\leq & C \phi \phi\left\|_{*}^{2}\left(\sum_{j=1}^{k} \frac{1}{\left(1+\left|y-\mathbf{x}_{j}\right|\right)^{\frac{N-2}{2}+\tau}}\right)^{2^{*-1}}+C\right\| \phi \|_{*}^{2^{*}-1} \sum_{j=1}^{k} \frac{1}{\left(1+\left|y-\mathbf{x}_{j}\right|\right)^{\frac{N+2}{2}+\tau}} \\
\leq & C\|\phi\|_{*}^{2} \sum_{j=1}^{k} \frac{1}{\left(1+\left|y-\mathbf{x}_{j}\right|\right)^{\frac{N+2}{2}+\tau}} .
\end{aligned}
$$

Next, we estimate $l_{k}$.

Lemma 2.6. We have

$$
\left\|l_{k}\right\|_{* *} \leq C\left(s \varepsilon^{\frac{N-2}{2}}\right)^{\frac{1}{2}+\sigma}+C|\lambda| \varepsilon^{1+\sigma},
$$

where $\sigma>0$ is a fixed small constant.

Proof. Recall

$$
\Omega_{j}=\left\{y=\left(y^{\prime}, y^{\prime \prime}\right) \in \Omega_{\varepsilon}:\left\langle\frac{y^{\prime}}{\left|y^{\prime}\right|}, \frac{\mathbf{x}_{j}}{\left|\mathbf{x}_{j}\right|}\right\rangle \geq \cos \frac{\pi}{k}\right\} .
$$

From the symmetry, we can assume that $y \in \Omega_{1}$. Then,

$$
\left|y-\mathbf{x}_{j}\right| \geq\left|y-\mathbf{x}_{1}\right|, \quad \forall y \in \Omega_{1} \text {. }
$$


Thus, for $y \in \Omega_{1}$, by Lemma A.1,

$$
\begin{aligned}
\left|l_{k}\right| \leq & \frac{C}{\left(1+\left|y-\mathbf{x}_{1}\right|\right)^{4-\beta}} \sum_{j=2}^{k} \frac{1}{\left(1+\left|y-\mathbf{x}_{j}\right|\right)^{N-2-\beta}} \\
& +C\left(\sum_{j=2}^{k} \frac{1}{\left(1+\left|y-\mathbf{x}_{j}\right|\right)^{N-2-\beta}}\right)^{2^{*}-1} \\
& +C \sum_{j=1}^{k} \frac{1}{\left(1+\left|y-\mathbf{x}_{j}\right|\right)^{4-\beta}}\left(\varepsilon^{N-2}+\frac{|\lambda| \varepsilon^{2}}{\left(1+\left|y-\mathbf{x}_{j}\right|\right)^{N-4-\beta}}\right) \\
& +C W_{r, \Lambda}^{2^{*}-1-\frac{1}{2}-\frac{2 \sigma}{N-2}} s^{\frac{1}{2}+\frac{2 \sigma}{N-2}} \varepsilon^{\frac{N-2}{4}+\sigma} .
\end{aligned}
$$

Here, we have used the inequality: for any bounded $a>0$ and $b>0, \alpha \in(0,1]$ :

$$
\left|(a-b)_{+}^{2^{*}-1}-a^{2^{*}-1}\right| \leq C a^{2^{*}-1-\alpha} b^{\alpha} .
$$

Let us estimate the first term of (2.22). Using Lemma B.2, we obtain

$$
\begin{aligned}
& \frac{1}{\left(1+\left|y-\mathbf{x}_{1}\right|\right)^{4-\beta}} \frac{1}{\left(1+\left|y-\mathbf{x}_{j}\right|\right)^{N-2-\beta}} \\
\leq & C\left(\frac{1}{\left(1+\left|y-\mathbf{x}_{1}\right|\right)^{\frac{N+2}{2}+\tau}}+\frac{1}{\left(1+\left|y-\mathbf{x}_{j}\right|\right)^{\frac{N+2}{2}+\tau}}\right) \frac{1}{\left|\mathbf{x}_{j}-\mathbf{x}_{1}\right|^{\frac{N+2}{2}-\tau-2 \beta}} \\
\leq & C \frac{1}{\left(1+\left|y-\mathbf{x}_{1}\right|\right)^{\frac{N+2}{2}+\tau}} \frac{1}{\left|\mathbf{x}_{j}-\mathbf{x}_{1}\right|^{\frac{N+2}{2}-\tau-2 \beta}}, \quad j>1 .
\end{aligned}
$$

Since $\frac{N+2}{2}-\tau-2 \beta>1$, we find

$$
\begin{aligned}
& \frac{1}{\left(1+\left|y-\mathbf{x}_{1}\right|\right)^{4-\beta}} \sum_{j=2}^{k} \frac{1}{\left(1+\left|y-\mathbf{x}_{j}\right|\right)^{N-2-\beta}} \\
\leq & C \frac{1}{\left(1+\left|y-\mathbf{x}_{1}\right|\right)^{\frac{N+2}{2}+\tau}}(k \varepsilon)^{\frac{N+2}{2}-\tau-2 \beta} \\
\leq & C\left(s \varepsilon^{\frac{N-2}{2}}\right)^{\frac{1}{2}+\sigma} \frac{1}{\left(1+\left|y-\mathbf{x}_{1}\right|\right)^{\frac{N+2}{2}+\tau}} .
\end{aligned}
$$

Here we have used

$$
(k \varepsilon)^{\frac{N+2}{2}-\tau-2 \beta}=O\left(\left(s \varepsilon^{\frac{N-2}{2}}\right)^{\frac{1}{2}+\sigma}\right),
$$

for some small $\sigma>0$. 

result,

In fact, if $s>0$ is fixed (as in Theorem 1.4), then $k=\frac{1}{\sqrt{\varepsilon}}$ and $\tau=\frac{1}{2}$. As a

$$
(k \varepsilon)^{\frac{N+2}{2}-\tau-2 \beta}=O\left(\varepsilon^{\frac{N+2}{4}-\frac{\tau}{2}-\beta}\right)=O\left(\varepsilon^{\frac{N-2}{4}+\sigma}\right) .
$$

So, we obtain (2.25).

If $N \geq 7$, then $\tau=\frac{1}{2}$, and

$$
s^{\frac{(2-2 \theta)(N-4)}{(N-6)(N-2)}} \leq k \leq s^{\frac{(2-\theta)(N-4)}{(N-6)(N-2)}} .
$$

But

$$
(k \varepsilon)^{\frac{N+2}{2}-\tau-2 \beta}=\left(\frac{s^{\frac{2}{N-2}}}{k}\right)^{\frac{N+2}{2}-\tau-2 \beta}=\frac{s^{\frac{N+1-4 \beta}{N-2}}}{k^{\frac{N+1-4 \beta}{2}}}
$$

and

$$
\left(s \varepsilon^{\frac{N-2}{2}}\right)^{\frac{1}{2}+\sigma}=\left(\frac{s^{2}}{k^{N-2}}\right)^{\frac{1}{2}+\sigma}
$$

Thus, we see that (2.25) is equivalent to

$$
s^{\frac{3-4 \beta}{N-2}-2 \sigma} \leq C k^{\frac{3}{2}-2 \beta-(N-2) \sigma} .
$$

Using (2.26), we find (2.27) holds.

For $N=3, k=\frac{s}{\sqrt{\varepsilon}}$. Thus,

$$
(k \varepsilon)^{\frac{5}{2}-\tau-2 \beta}=\left(s \varepsilon^{\frac{1}{2}}\right)^{\frac{5}{2}-\tau-2 \beta} \leq C\left(s \varepsilon^{\frac{1}{2}}\right)^{\frac{1}{2}+\sigma} .
$$

So, we obtain (2.25).

Now, we estimate the second term of (2.22).

Using Lemma B.2 again, we find for $y \in \Omega_{1}$,

$$
\begin{aligned}
& \frac{1}{\left(1+\left|y-\mathbf{x}_{j}\right|\right)^{N-2-\beta}} \leq \frac{1}{\left(1+\left|y-\mathbf{x}_{1}\right|\right)^{\frac{N-2-\beta}{2}}} \frac{1}{\left(1+\left|y-\mathbf{x}_{j}\right|\right)^{\frac{N-2-\beta}{2}}} \\
\leq & \frac{C}{\left|\mathbf{x}_{j}-\mathbf{x}_{1}\right|^{\frac{N-2}{2}-\beta-\frac{N-2}{N+2} \tau}}\left(\frac{1}{\left(1+\left|y-\mathbf{x}_{1}\right|\right)^{\frac{N-2}{2}+\frac{N-2}{N+2} \tau}}+\frac{1}{\left(1+\left|y-\mathbf{x}_{j}\right|\right)^{\frac{N-2}{2}+\frac{N-2}{N+2} \tau}}\right) \\
\leq & \frac{C}{\left|\mathbf{x}_{j}-\mathbf{x}_{1}\right|^{\frac{N-2}{2}-\beta-\frac{N-2}{N+2} \tau}} \frac{1}{\left(1+\left|y-\mathbf{x}_{1}\right|\right)^{\frac{N-2}{2}+\frac{N-2}{N+2} \tau}} .
\end{aligned}
$$


Suppose that $N \geq 5$. Then $\frac{N-2}{2}-\beta-\frac{N-2}{N+2} \tau>1$ since $\tau<1$. Then (2.28) gives for $y \in \Omega_{1}$

$$
\begin{aligned}
& \left(\sum_{j=2}^{k} \frac{1}{\left(1+\left|y-\mathbf{x}_{j}\right|\right)^{N-2-\beta}}\right)^{2^{*}-1} \\
\leq & C(k \varepsilon)^{\frac{N+2}{2}-\tau-\left(2^{*}-1\right) \beta} \frac{1}{\left(1+\left|y-\mathbf{x}_{1}\right|\right)^{\frac{N+2}{2}+\tau}} \\
= & C\left(s \varepsilon^{\frac{N-2}{2}}\right)^{\frac{1}{2}+\sigma} \frac{1}{\left(1+\left|y-\mathbf{x}_{1}\right|\right)^{\frac{N+2}{2}+\tau}} .
\end{aligned}
$$

If $N=3,4$, then (2.28) gives

$$
\begin{aligned}
& \left(\sum_{j=2}^{k} \frac{1}{\left(1+\left|y-\mathbf{x}_{j}\right|\right)^{N-2-\beta}}\right)^{2^{*}-1} \\
\leq & C\left(k \varepsilon^{\frac{N-2}{2}-\frac{N-2}{N+2} \tau-\beta}\right)^{2^{*}-1} \frac{1}{\left(1+\left|y-\mathbf{x}_{1}\right|\right)^{\frac{N+2}{2}+\tau}} \\
= & C k^{\frac{N+2}{N-2}} \varepsilon^{\frac{N+2}{2}-\tau-\left(2^{*}-1\right) \beta} \frac{1}{\left(1+\left|y-\mathbf{x}_{1}\right|\right)^{\frac{N+2}{2}+\tau}} .
\end{aligned}
$$

If $N=4$, then

$$
k^{\frac{N+2}{N-2}} \varepsilon^{\frac{N+2}{2}-\tau-\left(2^{*}-1\right) \beta}=k^{3} \varepsilon^{3-\frac{1}{2}-\left(2^{*}-1\right) \beta} \leq C \varepsilon^{1-\left(2^{*}-1\right) \beta} \leq C \varepsilon^{\frac{1}{2}+\sigma} .
$$

Hence for $N=4$,

$$
\left(\sum_{j=2}^{k} \frac{1}{\left(1+\left|y-\mathbf{x}_{j}\right|\right)^{2}}\right)^{2^{*}-1} \leq \sum_{i=1}^{k} \frac{C \varepsilon^{\frac{N-2}{4}+\sigma}}{\left(1+\left|y-\mathbf{x}_{i}\right|\right)^{\frac{N+2}{2}+\tau}} .
$$

For $N=3$, we have

$$
k^{5} \varepsilon^{\frac{5}{2}-\tau-\left(2^{*}-1\right) \beta}=k^{2 \tau+2\left(2^{*}-1\right) \beta} s^{5-2 \tau-2\left(2^{*}-1\right) \beta} .
$$

But

$$
\left(s \varepsilon^{\frac{1}{2}}\right)^{\frac{1}{2}+\sigma}=\frac{s^{1+2 \sigma}}{k^{\frac{1}{2}+\sigma}} .
$$


So, $k^{5} \varepsilon^{\frac{5}{2}-\tau-\left(2^{*}-1\right) \beta} \leq C\left(s \varepsilon^{\frac{1}{2}}\right)^{\frac{1}{2}+\sigma}$ is equivalent to

$$
k \leq C s^{-\frac{\left.8-4 \tau-4 \sigma-4\left(2^{*}-1\right) \beta\right)}{\left.1+4 \tau+2 \sigma+4\left(2^{*}-1\right) \beta\right)}}
$$

Since $k \leq C s^{-\frac{2 \tau}{1-2 \tau}}$, we see that (2.31) is valid if

$$
\frac{8-4 \tau}{1+4 \tau}>\frac{2 \tau}{1-2 \tau}
$$

Thus, if $\tau \in\left(0, \frac{4}{11}\right),(2.31)$ holds. Hence for $N=3$, we also have

$$
\left(\sum_{j=2}^{k} \frac{1}{\left(1+\left|y-\mathbf{x}_{j}\right|\right)^{2}}\right)^{2^{*}-1} \leq \sum_{i=1}^{k} \frac{C\left(s \varepsilon^{\frac{N-2}{2}}\right)^{\frac{1}{2}+\sigma}}{\left(1+\left|y-\mathbf{x}_{i}\right|\right)^{\frac{N+2}{2}+\tau}} .
$$

Note that for $y \in \Omega_{1}$,

$$
W_{r, \Lambda}(y) \leq \frac{C}{\left(1+\left|y-\mathbf{x}_{1}\right|\right)^{N-2-\tau-\beta}} .
$$

We claim that

$$
\left(\frac{N+2}{N-2}-\frac{1}{2}-\frac{2 \sigma}{N-2}\right)(N-2-\tau) \geq \frac{N+2}{2}+\tau,
$$

if $N \geq 3$.

In fact, (2.32) is equivalent to

$$
\tau<\frac{4(N-2)}{3 N+2},
$$

which is true, since $\tau=\frac{1}{2}$ if $N \geq 4, \tau<\frac{4}{11}$ if $N=3$.

Thus, we obtain

$$
s^{\frac{1}{2}+\frac{2 \sigma}{N-2}} \varepsilon^{\frac{N-2}{4}+\sigma} W_{r, \Lambda}^{\frac{N+2}{N-2}-\frac{1}{2}-\frac{2 \sigma}{N-2}} \leq C s^{\frac{1}{2}+\frac{2 \sigma}{N-2}} \varepsilon^{\frac{N-2}{4}+\sigma} \frac{C}{\left(1+\left|y-\mathbf{x}_{j}\right|\right)^{\frac{N+2}{2}+\tau}} .
$$

Finally,

$$
\begin{aligned}
\sum_{j=1}^{k} \frac{1}{\left(1+\left|y-\mathbf{x}_{j}\right|\right)^{4}} \frac{|\lambda| \varepsilon^{2}}{\left(1+\left|y-\mathbf{x}_{j}\right|\right)^{N-4-\beta}} & =\sum_{j=1}^{k} \frac{|\lambda| \varepsilon^{2}}{\left(1+\left|y-\mathbf{x}_{j}\right|\right)^{N-\beta}} \\
& \leq C|\lambda| \varepsilon^{2} \sum_{j=1}^{k} \frac{1}{\left(1+\left|y-\mathbf{x}_{j}\right|\right)^{\frac{N+2}{2}+\tau}},
\end{aligned}
$$


and

$$
\begin{aligned}
& \sum_{j=1}^{k} \frac{1}{\left(1+\left|y-\mathbf{x}_{j}\right|\right)^{4}} \varepsilon^{N-2} \leq C \varepsilon^{N-2-\frac{N-6}{2}-\tau} \sum_{j=1}^{k} \frac{1}{\left(1+\left|y-\mathbf{x}_{j}\right|\right)^{\frac{N+2}{2}+\tau}} \\
= & C \varepsilon^{\frac{N+2}{2}-\tau} \sum_{j=1}^{k} \frac{1}{\left(1+\left|y-\mathbf{x}_{j}\right|\right)^{\frac{N+2}{2}+\tau}} \leq C(k \varepsilon)^{\frac{N+2}{2}-\tau} \sum_{j=1}^{k} \frac{1}{\left(1+\left|y-\mathbf{x}_{j}\right|\right)^{\frac{N+2}{2}+\tau}} \\
\leq & C\left(s \varepsilon^{\frac{N-2}{2}}\right)^{\frac{1}{2}+\sigma} \sum_{j=1}^{k} \frac{1}{\left(1+\left|y-\mathbf{x}_{j}\right|\right)^{\frac{N+2}{2}+\tau}} .
\end{aligned}
$$

Combining all the above estimates, we obtain the result.

Now, we are ready to prove Proposition 2.4.

Proof of Proposition 2.4. Let us recall that

$$
\varepsilon=\frac{s^{\frac{2}{N-2}}}{k^{2}}
$$

Let

$$
E_{N}=\left\{u: u \in C\left(\Omega_{\varepsilon}\right),\|u\|_{*} \leq \sqrt{s} \varepsilon^{\frac{N-2}{4}}, \int_{\Omega_{\varepsilon}} \sum_{i=1}^{k} Z_{i, j} u=0, j=1,2\right\}
$$

Then, (2.19) is equivalent to

$$
\phi=A(\phi)=: L(\bar{N}(\phi))+L\left(l_{k}\right) .
$$

Now we prove that $A$ is a contraction map from $E_{N}$ to $E_{N}$. Using Lemma 2.5, we have

$$
\begin{aligned}
\|A \phi\|_{*} \leq C\|\bar{N}(\phi)\|_{* *}+C\left\|l_{k}\right\|_{* *} & \leq C\|\phi\|_{*}^{\min \left(2^{*}-1,2\right)}+C\left\|l_{k}\right\|_{* *} \\
& \leq C\left(\sqrt{s} \varepsilon^{\frac{N-2}{4}}\right)^{\min \left(2^{*}-1,2\right)}+C\left\|l_{k}\right\|_{* *} \\
& \leq C\left(\sqrt{s} \varepsilon^{\frac{N-2}{4}}\right)^{1+\sigma}+C\left\|l_{k}\right\|_{* *} .
\end{aligned}
$$

Thus, by Lemma 2.6, we find that $A$ maps $E_{N}$ to $E_{N}$.

Next, we show that $A$ is a contraction map.

$$
\left\|A\left(\phi_{1}\right)-A\left(\phi_{2}\right)\right\|_{*}=\left\|L\left(\bar{N}\left(\phi_{1}\right)\right)-L\left(\bar{N}\left(\phi_{2}\right)\right)\right\|_{*} \leq C\left\|\bar{N}\left(\phi_{1}\right)-\bar{N}\left(\phi_{2}\right)\right\|_{* *} .
$$

Using

$$
\left|\bar{N}^{\prime}(t)\right| \leq \begin{cases}C|t|^{2^{*}-2}, & N \geq 6 \\ C\left(W^{\frac{6-N}{N-2}}|\phi|+|\phi|^{2^{*}-2}\right), & N=3,4,5\end{cases}
$$


we can prove that

$$
\begin{aligned}
\left\|A\left(\phi_{1}\right)-A\left(\phi_{2}\right)\right\|_{*} & \leq C\left\|\bar{N}\left(\phi_{1}\right)-\bar{N}\left(\phi_{2}\right)\right\|_{* *} \\
& \leq C\left(\left\|\phi_{1}\right\|_{*}^{\min \left(1,2^{*}-2\right)}+\left\|\phi_{2}\right\|_{*}^{\min \left(1,2^{*}-2\right)}\right)\left\|\phi_{1}-\phi_{2}\right\|_{*} \\
& \leq \frac{1}{2}\left\|\phi_{1}-\phi_{2}\right\|_{*} .
\end{aligned}
$$

Thus, $A$ is a contraction map.

It follows from the contraction mapping theorem that there is a unique $\phi \in E_{N}$, such that

$$
\phi=A(\phi) .
$$

Moreover, it follows from (2.33) that

$$
\|\phi\|_{*} \leq C\left(\sqrt{s} \varepsilon^{\frac{N-2}{4}}\right)^{1+\sigma}+C\left\|l_{k}\right\|_{* *} .
$$

So, the estimate for $\|\phi\|_{*}$ follows from Lemma 2.6.

\section{Proof of the main results}

Let

$$
F(r, \Lambda)=I\left(W_{r, \Lambda}+\phi\right),
$$

where $\phi$ is the function obtained in Proposition 2.4, and let

$$
I(u)=\frac{1}{2} \int_{\Omega_{\varepsilon}}\left(|D u|^{2}-\lambda \varepsilon^{2} u^{2}\right)-\frac{1}{2^{*}} \int_{\Omega_{\varepsilon}}\left(u-s \Phi_{\varepsilon}\right)_{+}^{2^{*}} .
$$

Using the symmetry, we can check that if $(r, \Lambda)$ is a critical point of $F(\Lambda)$, then $W_{r, \Lambda}+\phi$ is a solution of (1.3).

Proposition 3.1. We have

$$
\begin{aligned}
F(r, \Lambda)=k\left(A_{0}+\right. & \frac{A_{2} \bar{\varphi}(r) s \varepsilon^{\frac{N-2}{2}}}{\Lambda^{\frac{N-2}{2}}}-\frac{A_{3} k^{N-2} \varepsilon^{N-2}}{r^{N-2} \Lambda^{N-2}} \\
& \left.+O\left(\left(s \varepsilon^{\frac{N-2}{2}}\right)^{1+\sigma}+(k \varepsilon)^{(N-2)(1+\sigma)}\right)\right), \quad N=3,4
\end{aligned}
$$

and

$$
\begin{aligned}
F(r, \Lambda)=k\left(A_{0}-\right. & \frac{A_{1} \lambda \varepsilon^{2}}{\Lambda^{2}}+\frac{A_{2} \bar{\varphi}(r) s \varepsilon^{\frac{N-2}{2}}}{\Lambda^{(N-2) / 2}}-\frac{A_{3} k^{N-2} \varepsilon^{N-2}}{r^{N-2} \Lambda^{N-2}} \\
& \left.+O\left(|\lambda| \varepsilon^{2+\sigma}+\left(s \varepsilon^{\frac{N-2}{2}}\right)^{1+\sigma}+(k \varepsilon)^{(N-2)(1+\sigma)}\right)\right), \quad N \geq 5 .
\end{aligned}
$$

where the constant $A_{i}>0, i=0,1,2$ are positive constants, which are given in Proposition A.3. 
Proof. There is $t \in(0,1)$, such that

$$
\begin{aligned}
& \quad F(r, \Lambda)=I\left(W_{r, \Lambda}\right)+\left\langle I^{\prime}\left(W_{r, \Lambda}\right), \phi\right\rangle+\frac{1}{2} D^{2} I\left(W_{r, \Lambda}+t \phi\right)(\phi, \phi) \\
& =I\left(W_{r, \Lambda}\right)-\int_{\Omega_{\varepsilon}} l_{k} \phi+\int_{\Omega_{\varepsilon}}\left(|D \phi|^{2}+\varepsilon^{2} \mu \phi^{2}-\left(2^{*}-1\right)\left(W_{r, \Lambda}-s \Phi_{\varepsilon}+t \phi\right)_{+}^{2^{*}-2} \phi^{2}\right) \\
& =I\left(W_{r, \Lambda}\right)-\left(2^{*}-1\right) \int_{\Omega_{\varepsilon}}\left(\left(W_{r, \Lambda}-s \Phi_{\varepsilon}+t \phi\right)_{+}^{2^{*}-2}-\left(W_{r, \Lambda}-s \Phi_{\varepsilon}\right)_{+}^{2^{*}-2}\right) \phi^{2} \\
& \quad+\int_{\Omega_{\varepsilon}} N(\phi) \phi \\
& =I\left(W_{r, \Lambda}\right)-\left(2^{*}-1\right) \int_{\Omega_{\varepsilon}}\left(\left(W_{r, \Lambda}-s \Phi_{\varepsilon}+t \phi\right)_{+}^{2^{*}-2}-\left(W_{r, \Lambda}-s \Phi_{\varepsilon}\right)_{+}^{2^{*}-2}\right) \phi^{2} \\
& \quad+O\left(\int_{\Omega_{\varepsilon}}|\bar{N}(\phi)||\phi|\right) .
\end{aligned}
$$

But

$$
\begin{aligned}
& \int_{\Omega_{\varepsilon}}|\bar{N}(\phi) \| \phi| \\
\leq & C\|\bar{N}(\phi)\|_{* *}\|\phi\|_{*} \int_{\Omega_{\varepsilon}} \sum_{j=1}^{k} \frac{1}{\left(1+\left|y-\mathbf{x}_{j}\right|\right)^{\frac{N+2}{2}+\tau}} \sum_{i=1}^{k} \frac{1}{\left(1+\left|y-\mathbf{x}_{i}\right|\right)^{\frac{N-2}{2}+\tau}} .
\end{aligned}
$$

Using Lemma B.2, we find

$$
\begin{aligned}
& \sum_{j=1}^{k} \frac{1}{\left(1+\left|y-\mathbf{x}_{j}\right|\right)^{\frac{N+2}{2}+\tau}} \sum_{i=1}^{k} \frac{1}{\left(1+\left|y-\mathbf{x}_{i}\right|\right)^{\frac{N-2}{2}+\tau}} \\
= & \sum_{j=1}^{k} \frac{1}{\left(1+\left|y-\mathbf{x}_{j}\right|\right)^{N+2 \tau}}+\sum_{j=1}^{k} \sum_{i \neq j} \frac{1}{\left(1+\left|y-\mathbf{x}_{j}\right|\right)^{\frac{N+2}{2}+\tau}} \frac{1}{\left(1+\left|y-\mathbf{x}_{i}\right|\right)^{\frac{N-2}{2}+\tau}} \\
\leq & \sum_{j=1}^{k} \frac{1}{\left(1+\left|y-\mathbf{x}_{j}\right|\right)^{N+2 \tau}}+C \sum_{j=1}^{k} \frac{1}{\left(1+\left|y-\mathbf{x}_{j}\right|\right)^{N+\frac{1}{2} \tau}} \sum_{i=2}^{k} \frac{1}{\left|\mathbf{x}_{i}-\mathbf{x}_{1}\right|^{\frac{3}{2} \tau}} \\
\leq & C \sum_{j=1}^{k} \frac{1}{\left(1+\left|y-\mathbf{x}_{j}\right|\right)^{N+\frac{1}{2} \tau}},
\end{aligned}
$$

Thus, we obtain

$\int_{\Omega_{\varepsilon}}\left|\bar{N}(\phi)\|\phi \mid \leq C k\| \bar{N}(\phi)\left\|_{* *}\right\| \phi\left\|_{*} \leq C k\right\| \phi \|_{*}^{2} \leq C k\left(|\lambda| \varepsilon^{2+\sigma}+\left(s \varepsilon^{\frac{N-2}{2}}\right)^{1+\sigma}\right)\right.$. 
Now

$$
\begin{aligned}
& \left(W_{r, \Lambda}-s \Phi_{\varepsilon}+t \phi\right)_{+}^{2^{*}-2}-\left(W_{r, \Lambda}-s \Phi_{\varepsilon}\right)_{+}^{2^{*}-2} \\
& = \begin{cases}O\left(|\phi|^{2^{*}-2}\right), & N \geq 6 ; \\
O\left(W_{r, \Lambda}^{\frac{6-N}{N-2}}|\phi|+|\phi|^{2^{*}-2}\right), & N=3,4,5 .\end{cases}
\end{aligned}
$$

Thus, we have

$$
\begin{gathered}
\left|\int_{\Omega_{\varepsilon}}\left(\left(W_{r, \Lambda}-s \Phi_{\varepsilon}+t \phi\right)^{2^{*}-2}\right)-\left(\left(W_{r, \Lambda}-s \Phi_{\varepsilon}\right)^{2^{*}-2}\right) \phi^{2}\right| \\
\leq C\|\phi\|_{*}^{2^{*}} \int_{\Omega_{\varepsilon}}\left(\sum_{j=1}^{k} \frac{1}{\left(1+\left|y-\mathbf{x}_{j}\right|\right)^{\frac{N-2}{2}+\tau}}\right)^{2^{*}},
\end{gathered}
$$

if $N \geq 6$. If $N=3,4,5$, noting that $N-2>\frac{N-2}{2}+\tau$, we obtain

$$
\begin{aligned}
& \left|\int_{\Omega_{\varepsilon}}\left(\left(W_{r, \Lambda}-s \Phi_{\varepsilon}+t \phi\right)^{2^{*}-2}\right)-\left(\left(W_{r, \Lambda}-s \Phi_{\varepsilon}\right)^{2^{*}-2}\right) \phi^{2}\right| \\
\leq & C \int_{\Omega_{\varepsilon}} W_{r, \Lambda}^{\frac{6-N}{N-2}}|\phi|^{3}+C \int_{\Omega_{\varepsilon}}|\phi|^{2^{*}} \leq\|\phi\|_{*}^{3} \int_{\Omega_{\varepsilon}}\left(\sum_{j=1}^{k} \frac{1}{\left(1+\left|y-\mathbf{x}_{j}\right|\right)^{\frac{N-2}{2}+\tau}}\right)^{2^{*}} .
\end{aligned}
$$

Let $\bar{\eta}>0$ be small. Using Lemma B.2, if $y \in \Omega_{1}$, then

$$
\begin{aligned}
& \sum_{j=2}^{k} \frac{1}{\left(1+\left|y-\mathbf{x}_{j}\right|\right)^{\frac{N-2}{2}+\tau}} \\
\leq & \sum_{j=2}^{k} \frac{1}{\left(1+\left|y-\mathbf{x}_{1}\right|\right)^{\frac{N-2}{4}+\frac{1}{2} \tau}} \frac{1}{\left(1+\left|y-\mathbf{x}_{j}\right|\right)^{\frac{N-2}{4}+\frac{1}{2} \tau}} \\
\leq & C \frac{1}{\left(1+\left|y-\mathbf{x}_{1}\right|\right)^{\frac{N-2}{2}+\frac{1}{2} \bar{\eta}}} \sum_{j=2}^{k} \frac{1}{\left|\mathbf{x}_{j}-\mathbf{x}_{1}\right|^{\tau-\frac{1}{2} \bar{\eta}}} \leq C \varepsilon^{-\bar{\eta}} \frac{1}{\left(1+\left|y-\mathbf{x}_{1}\right|\right)^{\frac{N-2}{2}+\frac{1}{2} \bar{\eta}}} .
\end{aligned}
$$

As a result,

$$
\left(\sum_{j=1}^{k} \frac{1}{\left(1+\left|y-\mathbf{x}_{j}\right|\right)^{\frac{N-2}{2}+\tau}}\right)^{2^{*}} \leq C \varepsilon^{-2^{*} \bar{\eta}} \frac{1}{\left(1+\left|y-\mathbf{x}_{1}\right|\right)^{N+2 * \frac{1}{2} \bar{\eta}}}, \quad y \in \Omega_{1} .
$$

Thus

$$
\int_{\Omega_{\varepsilon}}\left(\sum_{j=1}^{k} \frac{1}{\left(1+\left|y-\mathbf{x}_{j}\right|\right)^{\frac{N-2}{2}+\tau}}\right)^{2^{*}} \leq C k \varepsilon^{-2^{*} \bar{\eta}}
$$


So, we have proved

$$
\begin{aligned}
& \left|\int_{\Omega_{\varepsilon}}\left(\left(W_{r, \Lambda}-s \Phi_{\varepsilon}+t \phi\right)^{2^{*}-2}\right)-\left(\left(W_{r, \Lambda}-s \Phi_{\varepsilon}\right)^{2^{*}-2}\right) \phi^{2}\right| \\
\leq & C k \varepsilon^{-2^{*} \bar{\eta}}\|\phi\|_{*}^{\min \left(3,2^{*}\right)} \leq C k \varepsilon^{-2^{*} \bar{\eta}}\left(|\lambda| \varepsilon^{1+\sigma}+\left(s \varepsilon^{\frac{N-2}{2}}\right)^{\frac{1}{2}+\sigma}\right)^{\min \left(3,2^{*}\right)} \\
\leq & C k\left(|\lambda| \varepsilon^{2+\sigma}+\left(s \varepsilon^{\frac{N-2}{2}}\right)^{1+\sigma}\right)
\end{aligned}
$$

Combining (3.1), (3.2) and (3.3), we find

$$
F(r, \Lambda)=I\left(W_{r, \Lambda}\right)+k O\left(|\lambda| \varepsilon^{2+\sigma}+\left(s \varepsilon^{\frac{N-2}{2}}\right)^{1+\sigma}\right) .
$$

Proof of Theorems 1.4, 1.5 and 1.6. We just need to prove that $F(r, \Lambda)$ has a critical point. that

Firstly, we consider the cases $N \neq 6$. It follows from (3.4) and Proposition A.3

$$
\begin{aligned}
F(r, \Lambda)=k\left(A_{0}\right. & +\frac{A_{2} \bar{\varphi}(r) s \varepsilon^{\frac{N-2}{2}}}{\Lambda^{(N-2) / 2}}-\frac{A_{3} k^{N-2} \varepsilon^{N-2}}{r^{N-2} \Lambda^{N-2}} \\
& \left.+O\left((k \varepsilon)^{(N-2)(1+\sigma)}+\left(s \varepsilon^{\frac{N-2}{2}}\right)^{1+\sigma}\right)\right) .
\end{aligned}
$$

Let

$$
\bar{F}(r, \Lambda)=\frac{A_{2} \bar{\varphi}(r)}{\Lambda^{(N-2) / 2}}-\frac{A_{3}}{r^{N-2} \Lambda^{N-2}}, \quad(r, \Lambda) \in\left[r_{1}, r_{2}\right] \times\left[\delta, \delta^{-1}\right] .
$$

Then, $\bar{F}(r, \Lambda)$ has a maximum point at $\left(r_{0}, \Lambda_{0}\right)$, where

$$
\Lambda_{0}=\left(\frac{2 A_{3}}{A_{2} r_{0}^{N-2} \bar{\varphi}\left(r_{0}\right)}\right)^{\frac{2}{N-2}}
$$

and $r_{0}$ is a maximum point of $r^{\frac{N-2}{2}} \bar{\varphi}(r)=r^{\frac{N-2}{2}} \varphi_{1}(r, 0)$. So, if $\delta>0$ is small, $\left(r_{0}, \Lambda_{0}\right)$ is an interior point of $\left[r_{1}, r_{2}\right] \times\left[\delta, \delta^{-1}\right]$. Thus, if $k>0$ is large, $F(r, \Lambda)$ attains its maximum in the interior of $\left[r_{1}, r_{2}\right] \times\left[\delta, \delta^{-1}\right]$. As a result, $F(r, \Lambda)$ has a critical point in $\left[r_{1}, r_{2}\right] \times\left[\delta, \delta^{-1}\right]$.

If $N=6$, then

$$
\begin{gathered}
F(r, \Lambda)=k\left(A_{0}+\frac{-\lambda A_{1} \varepsilon^{2}+A_{2} \bar{\varphi}(r) s \varepsilon^{2}}{\Lambda^{2}}-\frac{A_{3} k^{4} \varepsilon^{4}}{r^{4} \Lambda^{4}}\right. \\
\left.+O\left((k \varepsilon)^{4(1+\sigma)}+\left(s \varepsilon^{2}\right)^{1+\sigma}\right)\right) .
\end{gathered}
$$


Let

$$
\bar{F}(r, \Lambda)=\frac{-\lambda A_{1} s^{-1}+A_{2} \bar{\varphi}(r)}{\Lambda^{2}}-\frac{A_{3}}{r^{4} \Lambda^{4}}, \quad(r, \Lambda) \in\left[r_{1}, r_{2}\right] \times\left[\delta, \delta^{-1}\right] .
$$

It is easy to see that there is an $s_{0}>0$, such that if $s>|\lambda| s_{0}$, then

$$
\tilde{\varphi}(r)=: r^{\frac{N-2}{2}}\left(-\lambda A_{1} s^{-1}+A_{2} \bar{\varphi}(r)\right), \quad r \in\left[r_{1}, r_{2}\right]
$$

has a maximum point $r_{0} \in\left(r_{1} . r_{2}\right)$ and $\tilde{\varphi}\left(r_{0}\right)>0$. Then, $\bar{F}(r, \Lambda)$ has a maximum point at $\left(r_{0}, \Lambda_{0}\right)$, where

$$
\Lambda_{0}=\left(\frac{2 A_{3}}{r_{0}^{4} \tilde{\varphi}\left(r_{0}\right)}\right)^{\frac{1}{2}}
$$

So, we can prove that $F(r, \Lambda)$ has a critical point in $\left[r_{1}, r_{2}\right] \times\left[\delta, \delta^{-1}\right]$.

\section{A. Appendix}

In this section, we will expand $I\left(W_{r, \Lambda}\right)$. We always assume that $d\left(\overline{\mathbf{x}}_{j}, \partial \Omega\right) \geq c_{0}>$ 0 , where $\overline{\mathbf{x}}_{j}=\varepsilon \mathbf{x}_{j}$. Denote

$$
\bar{\varphi}(r)=\varphi_{1}(r, 0) .
$$

First, let us recall that $W_{\Lambda, \xi}$ is the solution of

$$
\begin{cases}-\Delta W-\lambda \varepsilon^{2} W=U_{\Lambda, \xi}^{2^{*}-1} & \text { in } \Omega_{\varepsilon} \\ W=0 & \text { on } \partial \Omega_{\varepsilon}\end{cases}
$$

Let

$$
\psi_{\Lambda, \xi}=U_{\Lambda, \xi}-W_{\Lambda, \xi}
$$

Then,

$$
\begin{cases}-\Delta \psi_{\Lambda, \xi}-\lambda \varepsilon^{2} \psi_{\Lambda, \xi}=-\lambda \varepsilon^{2} U_{\Lambda, \xi} & \text { in } \Omega_{\varepsilon} \\ \psi_{\Lambda, \xi}=U_{\Lambda, \xi}, & \text { on } \partial \Omega_{\varepsilon}\end{cases}
$$

To calculate $I\left(W_{r, \Lambda}\right)$, we need to estimate $\psi_{\Lambda, \xi}$.

Decompose $\psi_{\Lambda, \xi}$ as follows

$$
\psi_{\Lambda, \xi}=\psi_{\Lambda, \xi, 1}+\psi_{\Lambda, \xi, 2}
$$

where $\psi_{\Lambda, \xi, 1}$ is the solution of

$$
\begin{cases}-\Delta \psi_{\Lambda, \xi, 1}-\lambda \varepsilon^{2} \psi_{\Lambda, \xi, 1}=-\lambda \varepsilon^{2} U_{\Lambda, \xi} & \text { in } \Omega_{\varepsilon} \\ \psi_{\Lambda, \xi}=0, & \text { on } \partial \Omega_{\varepsilon}\end{cases}
$$


and $\psi_{\Lambda, \xi, 2}$ is the solution of

$$
\begin{cases}-\Delta \psi_{\Lambda, \xi, 2}-\lambda \varepsilon^{2} \psi_{\Lambda, \xi, 2}=0, & \text { in } \Omega_{\varepsilon} \\ \psi_{\Lambda, \xi}=U_{\Lambda, \xi}, & \text { on } \partial \Omega_{\varepsilon}\end{cases}
$$

Since

$$
U_{\Lambda, \xi} \leq C \varepsilon^{N-2}, \quad \text { on } \partial \Omega_{\varepsilon}
$$

it is easy to see that

$$
\left|\psi_{\Lambda, \xi, 2}\right| \leq C \varepsilon^{N-2}
$$

Let $\bar{\psi}_{\Lambda, \xi, \varepsilon}$ be the solution of

$$
\begin{cases}-\Delta \psi-\lambda \varepsilon^{2} \psi=U_{\Lambda, \xi} & \text { in } \Omega_{\varepsilon} \\ \psi=0, & \text { on } \partial \Omega_{\varepsilon}\end{cases}
$$

Then, we can check that

$$
\left|\bar{\psi}_{\Lambda, \xi, \varepsilon}(y)\right| \leq \frac{C \ln ^{m}(2+|y-\xi|)}{(1+|y-\xi|)^{N-4}},
$$

where $m=1$ if $N=4$, otherwise, $m=0$. Thus, we have

Lemma A.1. We have

$$
\psi_{\Lambda, \xi}=-\lambda \varepsilon^{2} \bar{\psi}_{\lambda, \xi, \varepsilon}+O\left(\varepsilon^{N-2}\right)
$$

where $\bar{\psi}_{\lambda, \xi, \varepsilon}$ is the solution of (A.6). Moreover,

$$
\left|W_{\Lambda, \xi}\right| \leq C|\ln \varepsilon|^{m} U_{\Lambda, \xi}
$$

where $m=1$ if $N=4$, otherwise, $m=0$.

Proof. We only need to show

$$
\left|W_{\Lambda, \xi}\right| \leq C|\ln \varepsilon|^{m} U_{\Lambda, \xi}
$$

which follows from (A.7) and $\varepsilon \leq \frac{C}{1+|y-\xi|}$.

Proposition A.2. We have

$$
I\left(W_{\Lambda, \mathbf{x}_{j}}\right)=A_{0}+\frac{A_{2} \bar{\varphi}(r) s \varepsilon^{\frac{N-2}{2}}}{\Lambda^{\frac{N-2}{2}}}+O\left(\left(s \varepsilon^{\frac{N-2}{2}}\right)^{1+\sigma}\right), \quad N=3,4,
$$

and

$$
I\left(W_{\Lambda, \mathbf{x}_{j}}\right)=A_{0}-\frac{A_{1} \lambda \varepsilon^{2}}{\Lambda^{2}}+\frac{A_{2} \bar{\varphi}(r) s \varepsilon^{\frac{N-2}{2}}}{\Lambda^{\frac{N-2}{2}}}+O\left(|\lambda| \varepsilon^{2+\sigma}+\left(s \varepsilon^{\frac{N-2}{2}}\right)^{1+\sigma}\right), \quad N \geq 5
$$


where

$$
\begin{gathered}
A_{0}=\frac{1}{2} \int_{\mathbb{R}^{N}}|D U|^{2}-\frac{1}{2^{*}} \int_{\mathbb{R}^{N}} U^{2^{*}}, \quad A_{2}=\int_{\mathbb{R}^{N}} U^{2^{*}-1}, \\
A_{1}=\frac{1}{2} \int_{\mathbb{R}^{N}} U^{2}, \quad N \geq 5,
\end{gathered}
$$

and $\sigma$ is some positive constant.

Proof. Write

$$
I(u)=\tilde{I}(u)-\frac{1}{2^{*}} \int_{\Omega_{\varepsilon}}\left(\left(u-s \Phi_{\varepsilon}\right)_{+}^{2^{*}}-|u|^{2^{*}}\right),
$$

where

$$
\tilde{I}(u)=\frac{1}{2} \int_{\Omega_{\varepsilon}}|D u|^{2}-\frac{1}{2} \lambda \varepsilon^{2} \int_{\Omega_{\varepsilon}} u^{2}-\frac{1}{2^{*}} \int_{\Omega_{\varepsilon}}|u|^{2^{*}} .
$$

By Lemma A.1, we have

$$
\begin{aligned}
\tilde{I}\left(W_{\Lambda, \mathbf{x}_{j}}\right) & =\frac{1}{2} \int_{\Omega_{\varepsilon}} U_{\Lambda, \mathbf{x}_{j}}^{2^{*}-1} W_{\Lambda, \mathbf{x}_{j}}-\frac{1}{2^{*}} \int_{\Omega_{\varepsilon}} W_{\Lambda, \mathbf{x}_{j}}^{2^{*}} \\
& =A_{0}+\frac{1}{2} \int_{\Omega_{\varepsilon}} U_{\Lambda, \mathbf{x}_{j}}^{2^{*}-1} \psi_{\Lambda, \mathbf{x}_{j}}+O\left(\int_{\Omega_{\varepsilon}} U_{\Lambda, \mathbf{x}_{j}}^{2^{*}-1-\sigma} \psi_{\Lambda, \mathbf{x}_{j}}^{1+\sigma}\right) \\
& =A_{0}+\frac{1}{2} \int_{\Omega_{\varepsilon}} U_{\Lambda, \mathbf{x}_{j}}^{2^{*}-1} \psi_{\Lambda, \mathbf{x}_{j}}+O\left(|\lambda| \varepsilon^{2(1+\sigma)}+\varepsilon^{(N-2)(1+\sigma)}\right) .
\end{aligned}
$$

On the other hand,

$$
\begin{aligned}
& \int_{\Omega_{\varepsilon}}\left(W_{\Lambda, \mathbf{x}_{j}}-s \Phi_{\varepsilon}\right)_{+}^{2^{*}}-\int_{\Omega_{\varepsilon}}\left(W_{\Lambda, \mathbf{x}_{j}}\right)^{2^{*}} \\
= & -2^{*} \int_{R^{N}} U^{2^{*}-1} s \varepsilon^{\frac{N-2}{2}} \Lambda_{j}^{-\frac{N-2}{2}} \bar{\varphi}(r)+O\left(\left(s \varepsilon^{\frac{N-2}{2}}\right)^{1+\sigma}\right) .
\end{aligned}
$$

For $N=3$, 4, by Lemma A.1 and (A.7),

$$
\int_{\Omega_{\varepsilon}} U_{\Lambda, \mathbf{x}_{j}}^{2^{*}-1} \psi_{\Lambda, \mathbf{x}_{j}}=O\left(\varepsilon^{N-2}+\varepsilon^{2}\right)=O\left(\left(s \varepsilon^{\frac{N-2}{2}}\right)^{1+\sigma}\right) .
$$

Here, we have used $\varepsilon=\frac{s^{2}}{k^{2}}=\frac{1}{k} s \sqrt{\varepsilon}=(s \sqrt{\varepsilon})^{1+\sigma}$ if $N=3$. So, the result for $N=3$, 4 follows from (A.8)-(A.10).

Suppose that $N \geq 5$. Let $\bar{\psi}_{\Lambda, \xi}$ be the solution of

$$
\begin{cases}-\Delta \psi=U_{\Lambda, \xi} & \text { in } \quad R^{N} \\ \psi(|y|) \rightarrow 0, & \text { as }|y| \rightarrow+\infty\end{cases}
$$


Then,

$$
\left|\bar{\psi}_{\Lambda, \xi}\right| \leq \frac{C}{(1+|y-\xi|)^{N-4}},
$$

and

$$
\left|\bar{\psi}_{\Lambda, \xi}-\bar{\psi}_{\Lambda, \xi, \varepsilon}\right| \leq \frac{C \varepsilon^{2} \ln ^{m}(2+|y-\xi|)}{(1+|y-\xi|)^{N-6}},
$$

where $m=1$ if $N=6$, otherwise, $m=0$. Thus,

$$
\begin{aligned}
\int_{\Omega_{\varepsilon}} U_{\Lambda, \mathbf{x}_{j}}^{2^{*}-1} \psi_{\Lambda, \mathbf{x}_{j}} & =-\lambda \varepsilon^{2} \int_{\mathbb{R}^{N}} U_{\Lambda, \mathbf{x}_{j}}^{2^{*}-1} \bar{\psi}_{\Lambda, \mathbf{x}_{j}}+O\left(\varepsilon^{N-2}+|\lambda| \varepsilon^{4}|\ln \varepsilon|\right) \\
& =-\lambda \varepsilon^{2} \int_{\mathbb{R}^{N}} U^{2}+O\left(\varepsilon^{N-2}+|\lambda| \varepsilon^{4}|\ln \varepsilon|\right) .
\end{aligned}
$$

So we obtain the result for $N \geq 5$.

Proposition A.3. We have

$$
\begin{aligned}
I\left(W_{r, \Lambda}\right)=k\left(A_{0}\right. & +\frac{A_{2} \bar{\varphi}(r) s \varepsilon^{\frac{N-2}{2}}}{\Lambda^{\frac{N-2}{2}}}-\frac{A_{3} k^{N-2} \varepsilon^{N-2}}{r \Lambda^{N-2}} \\
& \left.+O\left((k \varepsilon)^{(N-2)(1+\sigma)}+\left(s \varepsilon^{\frac{N-2}{2}}\right)^{1+\sigma}\right)\right), \quad N=3,4
\end{aligned}
$$

and

$$
\begin{aligned}
I\left(W_{r, \lambda}\right)=k\left(A_{0}\right. & -\frac{A_{1} \lambda \varepsilon^{2}}{\Lambda^{2}}+\frac{A_{2} \bar{\varphi}(r) s \varepsilon^{\frac{N-2}{2}}}{\Lambda^{(N-2) / 2}}-\frac{A_{3} k^{N-2} \varepsilon^{N-2}}{r^{N-2} \Lambda^{N-2}} \\
& \left.+O\left((k \varepsilon)^{(N-2)(1+\sigma)}+|\lambda| \varepsilon^{2+\sigma}+\left(s \varepsilon^{\frac{N-2}{2}}\right)^{1+\sigma}\right)\right), \quad N \geq 5 .
\end{aligned}
$$

Proof. By using the symmetry, we have

$$
\begin{gathered}
\int_{\Omega_{\varepsilon}}\left|D W_{r, \Lambda}\right|^{2}-\lambda \varepsilon^{2} \int_{\Omega_{\varepsilon}} W_{r, \Lambda}^{2}=\sum_{j=1}^{k} \sum_{i=1}^{k} \int_{\Omega_{\varepsilon}} U_{\Lambda, \mathbf{x}_{i}}^{2^{*}-1} W_{\Lambda, \mathbf{x}_{j}} \\
=k\left(\int_{\Omega_{\varepsilon}} U_{\Lambda, \mathbf{x}_{1}}^{2^{*}}+\int_{\Omega_{\varepsilon}} U_{\Lambda, \mathbf{x}_{1}}^{2^{*}-1} \psi_{\Lambda, \mathbf{x}_{1}}+\sum_{i=2}^{k} \int_{\Omega_{\varepsilon}} U_{\Lambda, \mathbf{x}_{1}}^{2^{*}-1} U_{\Lambda, \mathbf{x}_{i}}\right. \\
\left.+O\left(\sum_{i=2}^{k} \frac{1}{\left|\mathbf{x}_{i}-\mathbf{x}_{1}\right|^{N-2+\sigma}}\right)\right) \\
=k\left(\int_{\mathbb{R}^{N}} U^{2^{*}}+\int_{\Omega_{\varepsilon}} U_{\Lambda, \mathbf{x}_{1}}^{2^{*}-1} \psi_{\Lambda, \mathbf{x}_{1}}+\sum_{i=2}^{k} \frac{B_{0}}{\Lambda^{N-2}\left|\mathbf{x}_{i}-\mathbf{x}_{1}\right|^{N-2}}\right. \\
\left.+O\left(\sum_{i=2}^{k} \frac{1}{\left|\mathbf{x}_{i}-\mathbf{x}_{1}\right|^{N-2+\sigma}}\right)\right),
\end{gathered}
$$


where $B_{0}>0$ is a constant.

Let

$$
\Omega_{j}=\left\{y=\left(y^{\prime}, y^{\prime \prime}\right) \in \Omega_{\varepsilon}:\left\langle\frac{y^{\prime}}{\left|y^{\prime}\right|}, \frac{\mathbf{x}_{j}}{\left|\mathbf{x}_{j}\right|}\right\rangle \geq \cos \frac{\pi}{k}\right\} .
$$

Then,

$$
\left|y-\mathbf{x}_{i}\right| \geq\left|y-\mathbf{x}_{j}\right|, \quad \forall y \in \Omega_{j}
$$

We have

$$
\begin{aligned}
& \frac{1}{2^{*}} \int_{\Omega_{\varepsilon}}\left(W_{r, \Lambda}-s \Phi_{\varepsilon}\right)_{+}^{2^{*}}=\frac{k}{2^{*}} \int_{\Omega_{1}}\left(W_{r, \Lambda}-s \Phi_{\varepsilon}\right)_{+}^{2^{*}} \\
& =\frac{k}{2^{*}}\left(\int_{\Omega_{1}}\left(W_{\Lambda, \mathbf{x}_{1}}-s \Phi_{\varepsilon}\right)_{+}^{2^{*}}+2^{*} \int_{\Omega_{1}} \sum_{i=2}^{k}\left(W_{\Lambda, \mathbf{x}_{1}}-s \Phi_{\varepsilon}\right)_{+}^{2^{*}-1} W_{\Lambda, \mathbf{x}_{i}}\right. \\
& \left.+O\left(\int_{\Omega_{1}} W_{\Lambda, \mathbf{x}_{1}}^{2^{*}-2}\left(\sum_{i=2}^{k} W_{\Lambda, \mathbf{x}_{i}}\right)^{2}\right)\right) \\
& =\frac{k}{2^{*}}\left(\int_{\mathbb{R}^{N}} U^{2^{*}}-2^{*} \int_{\Omega_{\varepsilon}} U_{\Lambda, \mathbf{x}_{1}}^{2^{*}-1} \psi_{\Lambda, \mathbf{x}_{1}}-\frac{2^{*} A_{2} \bar{\varphi}(r) s \varepsilon^{\frac{N-2}{2}}}{\Lambda^{(N-2) / 2}}\right. \\
& +2^{*} \int_{\Omega_{1}} \sum_{i=2}^{k} U_{\Lambda, \mathbf{x}_{1}}^{2^{*}-1} U_{\Lambda, \mathbf{x}_{i}}+O\left(\int_{\Omega_{1}} U_{\Lambda, \mathbf{x}_{1}}^{2^{*}-2} s \Phi_{\varepsilon} \sum_{i=2}^{k} U_{\Lambda, \mathbf{x}_{i}}\right. \\
& \left.\left.+\int_{\Omega_{1}} U_{\Lambda, \mathbf{x}_{1}}^{2^{*}-2}\left(\sum_{i=2}^{k} U_{\Lambda, \mathbf{x}_{i}}\right)^{2}+\left(s \varepsilon^{\frac{N-2}{2}}\right)^{1+\sigma}+|\lambda| \varepsilon^{2+\sigma}\right)\right) \\
& =\frac{k}{2^{*}}\left(\int_{\mathbb{R}^{N}} U^{2^{*}}-2^{*} \int_{\Omega_{\varepsilon}} U_{\Lambda, \mathbf{x}_{1}}^{2^{*}-1} \psi_{\Lambda, \mathbf{x}_{1}}-\frac{2^{*} A_{2} \bar{\varphi}(r) s \varepsilon^{\frac{N-2}{2}}}{\Lambda^{\frac{N-2}{2}}}\right. \\
& +\sum_{i=2}^{k} \frac{2^{*} B_{0}}{\Lambda^{N-2}\left|\mathbf{x}_{i}-\mathbf{x}_{1}\right|^{N-2}} \\
& \left.+O\left((k \varepsilon)^{(N-2)(1+\sigma)}+\left(s \varepsilon^{\frac{N-2}{2}}\right)^{1+\sigma}+|\lambda| \varepsilon^{2+\sigma}\right)\right) \text {. }
\end{aligned}
$$

Since

$$
\left|\mathbf{x}_{j}-\mathbf{x}_{1}\right|=2\left|\mathbf{x}_{1}\right| \sin \frac{2(j-1) \pi}{k}, \quad j=2, \ldots, k
$$

we can prove

$$
\sum_{j=2}^{k} \frac{1}{\left|\mathbf{x}_{j}-\mathbf{x}_{1}\right|^{N-2}}=B_{4}(\varepsilon k)^{N-2}+O\left((k \varepsilon)^{(1+\sigma)(N-2)}\right) .
$$

Thus, the result follows from (A.13), (A.14) and (A.15). 


\section{B. Appendix}

Firstly, we gives a few lemmas, whose proof can be found in [35,37].

Lemma B.1. For any $\alpha>0$,

$$
\sum_{j=1}^{k} \frac{1}{\left(1+\left|y-\mathbf{x}_{j}\right|\right)^{\alpha}} \leq C\left(1+\sum_{j=2}^{k} \frac{1}{\left|\mathbf{x}_{1}-\mathbf{x}_{j}\right|^{\alpha}}\right),
$$

where $C>0$ is a constant, independent of $k$.

For each fixed $i$ and $j, i \neq j$, consider the following function

$$
g_{i j}(y)=\frac{1}{\left(1+\left|y-\mathbf{x}_{j}\right|\right)^{\alpha}} \frac{1}{\left(1+\left|y-\mathbf{x}_{i}\right|\right)^{\beta}},
$$

where $\alpha \geq 1$ and $\beta \geq 1$ are two constants. Then, we have

Lemma B.2. For any constant $0 \leq \sigma \leq \min (\alpha, \beta)$, there is a constant $C>0$, such that

$$
g_{i j}(y) \leq \frac{C}{\left|\mathbf{x}_{i}-\mathbf{x}_{j}\right|^{\sigma}}\left(\frac{1}{\left(1+\left|y-\mathbf{x}_{i}\right|\right)^{\alpha+\beta-\sigma}}+\frac{1}{\left(1+\left|y-\mathbf{x}_{j}\right|\right)^{\alpha+\beta-\sigma}}\right) .
$$

Lemma B.3. For any constant $0<\sigma<N-2$, there is a constant $C>0$, such that

$$
\int_{\mathbb{R}^{N}} \frac{1}{|y-z|^{N-2}} \frac{1}{(1+|z|)^{2+\sigma}} d z \leq \frac{C}{(1+|y|)^{\sigma}} .
$$

Let us recall that

$$
\varepsilon=\frac{s^{\frac{2}{N-2}}}{k^{2}} .
$$

For the constant $\tau \in(0,1)$ defined is (2.4),

$$
\sum_{j=2}^{k} \frac{1}{\left|\mathbf{x}_{j}-\mathbf{x}_{1}\right|^{\tau}} \leq C \varepsilon^{\tau} k^{\tau} \sum_{j=2}^{k} \frac{1}{j^{\tau}} \leq C \varepsilon^{\tau} k \leq C,
$$

and for any $\theta>0$,

$$
\sum_{j=2}^{k} \frac{1}{\left|\mathbf{x}_{j}-\mathbf{x}_{1}\right|^{\tau+\theta}}=o(1)
$$


Lemma B.4. Suppose that $N \geq 4$. There is a small $\theta>0$, such that

$$
\begin{aligned}
& \int_{\mathbb{R}^{N}} \frac{1}{|y-z|^{N-2}} W_{r, \Lambda}^{\frac{4}{N-2}}(z) \sum_{j=1}^{k} \frac{1}{\left(1+\left|z-\mathbf{x}_{j}\right|\right)^{\frac{N-2}{2}+\tau}} d z \\
\leq & C \sum_{j=1}^{k} \frac{1}{\left(1+\left|y-\mathbf{x}_{j}\right|\right)^{\frac{N-2}{2}+\tau+\theta}},
\end{aligned}
$$

where $W_{r, \Lambda}$ is defined in (1.7).

Proof. Recall that

$$
\Omega_{j}=\left\{y=\left(y^{\prime}, y^{\prime \prime}\right) \in \Omega_{\varepsilon}:\left\langle\frac{y^{\prime}}{\left|y^{\prime}\right|}, \frac{\mathbf{x}_{j}}{\left|\mathbf{x}_{j}\right|}\right\rangle \geq \cos \frac{\pi}{k}\right\} .
$$

For $z \in \Omega_{1}$, we have $\left|z-\mathbf{x}_{j}\right| \geq\left|z-\mathbf{x}_{1}\right|$. Using Lemma B.2, we obtain

$$
\begin{aligned}
\sum_{j=2}^{k} \frac{1}{\left(1+\left|z-\mathbf{x}_{j}\right|\right)^{N-2-\beta}} & \leq \frac{1}{\left(1+\left|z-\mathbf{x}_{1}\right|\right)^{\frac{1}{2}(N-2-\beta)}} \sum_{j=2}^{k} \frac{1}{\left(1+\left|z-\mathbf{x}_{j}\right|\right)^{\frac{1}{2}(N-2-\beta)}} \\
& \leq \frac{C}{\left(1+\left|z-\mathbf{x}_{1}\right|\right)^{N-2-\beta-\tau}} \sum_{j=2}^{k} \frac{1}{\left|\mathbf{x}_{j}-\mathbf{x}_{1}\right|^{\tau}} \\
& \leq \frac{C}{\left(1+\left|z-\mathbf{x}_{1}\right|\right)^{N-2-\beta-\tau}}
\end{aligned}
$$

Thus,

$$
W_{r, \Lambda}^{\frac{4}{N-2}}(z) \leq \frac{C}{\left(1+\left|z-\mathbf{x}_{1}\right|\right)^{4-\frac{4(\tau+\beta)}{N-2}}}
$$

As a result, for $z \in \Omega_{1}$, using Lemma B.2 again, we find that for $\theta>0$ small,

$$
W_{r, \Lambda}^{\frac{4}{N-2}}(z) \sum_{j=1}^{k} \frac{1}{\left(1+\left|z-\mathbf{x}_{j}\right|\right)^{\frac{N-2}{2}+\tau}} \leq \frac{C}{\left(1+\left|z-\mathbf{x}_{1}\right|\right)^{2+\frac{N-2}{2}+\tau+2-\tau-\frac{4(\tau+\beta)}{N-2}}}
$$

Since $\theta=: 2-\tau-\frac{4(\tau+\beta)}{N-2}>0$ if $N \geq 4$ and $\beta>0$ is small, we obtain

$$
\begin{aligned}
& \int_{\Omega_{1}} \frac{1}{|y-z|^{N-2}} W_{r, \Lambda}^{\frac{4}{N-2}}(z) \sum_{j=1}^{k} \frac{1}{\left(1+\left|z-\mathbf{x}_{j}\right|\right)^{\frac{N-2}{2}+\tau}} d z \\
\leq & \int_{\Omega_{1}} \frac{1}{|y-z|^{N-2}} \frac{C}{\left(1+\left|z-\mathbf{x}_{1}\right|\right)^{2+\frac{N-2}{2}+\tau+\theta}} d z \leq \frac{C}{\left(1+\left|y-\mathbf{x}_{1}\right|\right)^{\frac{N-2}{2}+\tau+\theta}},
\end{aligned}
$$


which gives

$$
\begin{aligned}
& \int_{\Omega_{\varepsilon}} \frac{1}{|y-z|^{N-2}} W_{r, \Lambda}^{\frac{4}{N-2}}(z) \sum_{j=1}^{k} \frac{1}{\left(1+\left|z-\mathbf{x}_{j}\right|\right)^{\frac{N-2}{2}+\tau}} d z \\
= & \sum_{i=1}^{k} \int_{\Omega_{i}} \frac{1}{|y-z|^{N-2}} W_{r, \Lambda}^{\frac{4}{N-2}}(z) \sum_{j=1}^{k} \frac{1}{\left(1+\left|z-\mathbf{x}_{j}\right|\right)^{\frac{N-2}{2}+\tau}} d z \\
\leq & \sum_{i=1}^{k} \frac{C}{\left(1+\left|y-\mathbf{x}_{i}\right|\right)^{\frac{N-2}{2}+\tau+\theta}} .
\end{aligned}
$$

The above proof does not work for $N=3$ because

$$
2-\tau-\frac{4 \tau}{N-2}<0
$$

if $N=3$ and $\tau=\frac{1}{2}$. The choice of $\tau \in(0,1)$ should ensure

$$
\sum_{j=2}^{k} \frac{1}{\left|\mathbf{x}_{j}-\mathbf{x}_{1}\right|^{\tau}} \leq C \varepsilon^{\tau} k \leq C .
$$

The above relation shows that $\tau$ can be chosen smaller if $\varepsilon$ becomes smaller, which in turn will make $2-\tau-\frac{4 \tau}{N-2}>0$. Noting that $\varepsilon=\frac{s^{2}}{k^{2}}$, we find that if $s \rightarrow 0+$, then $\varepsilon=o\left(\frac{1}{k^{2}}\right)$. We have

Lemma B.5. Suppose that $N=3$, the parameter $s>0$ and the integer $k$ satisfy

$$
s \leq C k^{-\frac{1}{2 \tau}+1}
$$

for some $\tau \in\left(0, \frac{2}{5}\right)$. Then, there is a small $\theta>0$, such that

$$
\begin{aligned}
& \int_{\mathbb{R}^{3}} \frac{1}{|y-z|} W_{r, \Lambda}^{4}(z) \sum_{j=1}^{k} \frac{1}{\left(1+\left|z-\mathbf{x}_{j}\right|\right)^{\frac{1}{2}+\tau}} d z \\
& \leq C \sum_{j=1}^{k} \frac{1}{\left(1+\left|y-\mathbf{x}_{j}\right|\right)^{\frac{1}{2}+\tau+\theta}} .
\end{aligned}
$$

Proof. The proof of this lemma is similar to that of Lemma B.4. We only need to use that for $\tau<\frac{2}{5}$,

$$
2-5 \tau>0
$$

and

$$
\varepsilon^{\tau} k=s^{2 \tau} k^{1-2 \tau} \leq C .
$$

Thus, we omit the details. 


\section{References}

[1] J. AI, K. S. CHOU and J. WEI, Self-similar solutions for the anisotropic affine curve shortening problem, Calc. Var. Partial Differential Equations 13 (2001), 311-337.

[2] Adimurthi and S. PRAShanth, Critical exponent problem in $\mathbb{R}^{2}$-border-line between existence and non-existence of positive solutions for Dirichlet problem, Adv. Differential Equations 5 (2000), 67-95.

[3] A. Ambrosetti and G. Prodi, On the inversion of some differentiable mappings with singularities between Banach spaces, Ann. Mat. Pura Appl. 93 (1973), 231-247.

[4] A. BAhri, "Critical Points at Infinity in Some Variational Problems", Research Notes in Mathematics, Vol. 182, Longman-Pitman, 1989.

[5] A. BAHRI, Y. Y. LI and O. REY, On a variational problem with lack of compactness: the topological effect of the critical points at infinity, Calc. Var. Partial Differential Equations 3 (1995), 67-93.

[6] B. Breuer, P. J. MCKennA and M. Plum, Multiple solutions for a semilinear boundary value problem: a computational multiplicity proof, J. Differential Equations 195 (2003), 243-269.

[7] H. Brezis and L. Nirenberg, Positive solutions of nonlinear elliptic equations involving critical Sobolev exponents, Comm. Pure Appl. Math. 36 (1983), 437-477.

[8] M. CALANCHI and B. RUF, Elliptic equations with one-sided critical growth, Electron. J. Differential Equations (2002), 1-21.

[9] E. N. DANCER, A Counter example to the Lazer-McKenna conjecture, Nonlinear Anal. 13 (1989), 19-21.

[10] E. N. DANCER and S. SANTRA, On the superlinear Lazer-McKenna conjecture: the nonhomogeneous case, Adv. Differential Equations 12(2007), 961-993.

[11] E. N. DANCER and S. YAN, On the superlinear Lazer-McKenna conjecture, J. Differential Equations 210 (2005), 317-351.

[12] E. N. DANCER and S. YAN, On the superlinear Lazer-McKenna conjecture, part two, Comm. Partial Differential Equations 30 (2005), 1331-1358.

[13] E. N. DANCER and S. YAN, The Lazer-McKenna conjecture and a free boundary problem in two dimensions, J. Lond. Math. Soc. 78 (2008), 639-662.

[14] M. Del Pino, P. Felmer and M. Musso, Two-bubble solutions in the super-critical Bahri-Coron's problem, Calc. Var. Partial Differential Equations 16 (2003), 113-145.

[15] M. Del Pino, M. Musso and A. Pistoia, Super-critical boundary bubbling in a semilinear Neumann problem, Ann. Inst. H. Poincaré Anal. Non Linéaire 22 (2005), 45-82.

[16] M. Del Pino, J. Dolbeault and M. Musso, The Brezis-Nirenberg problem near criticality in dimension 3, J. Math. Pures Appl. (9) 83 (2004), 1405-1456.

[17] M. DEL PINO and C. MunOZ, The two dimensional Lazer-McKenna conjecture for an exponential nonlinearity, J. Differential Equations 231 (2006), 108-134.

[18] D. G. De Figueiredo, On the superlinear Ambrosetti-Prodi problem, Nonlinear Anal. 8 (1984), 655-665.

[19] D. G. De Figueiredo and S. Solimini, A variational approach to superlinear elliptic problems, Comm. Partial Differential Equations 9 (1984), 699-717.

[20] D. G. De Figueiredo and J. YANG, Critical superlinear Ambrosetti-Prodi problems, Topol. Methods Nonlinear Anal. 14 (1999), 59-80.

[21] O. DRUET, The critical Lazer-McKenna conjecture in low dimensions, J. Differential Equations 231 (2008), 108-134.

[22] Y. GE, R. JING and F. PACARD, Bubble towers for supercritical semilinear elliptic equations, J. Funct. Anal. 221 (2005), 251-302.

[23] H. Hofer, Variational and topological methods in partial ordered Hilbert spaces, Math. Ann. 261 (1982), 493-514.

[24] A. C. LAZER and P. J. MCKenna, On the number of solutions of a nonlinear Dirichlet problem, J. Math. Anal. Appl. 84 (1981), 282-294. 
[25] A. C. LAZER and P. J. MCKENNA, On a conjecture related to the number of solutions of a nonlinear Dirichlet problem, Proc. Roy. Soc. Edinburgh 95A (1983), 275-283.

[26] A. C. LAZER and P. J. MCKenNA, A symmetric theorem and application to nonlinear partial differential equations, J. Differential Equations 72 (1988), 95-106.

[27] G. LI, S. YAN and J. YANG, The superlinear Lazer-McKenna onjecture for an elliptic problem with critical growth, Calc. Var. Partial Differential Equations 28 (2007), 471-508.

[28] G. LI, S. YAN and J. YANG, The superlinear Lazer-McKenna onjecture for an elliptic problem with critical growth, part II, J. Differential Equations 227 (2006), 301-332.

[29] O. REY, The role of the Green's function in a non-linear elliptic equation involving the critical Sobolev exponent, J. Funct. Anal. 89 (1990), 1-52.

[30] O. REY and J. WEI, Arbitrary number of positive solutions for an elliptic problem with critical nonlinearity, J. Eur. Math. Soc. (JEMS) 7 (2005), 449-476.

[31] B. RUF and S. SoliminI, On a class of superlinear Sturm-Liouville problems with arbitrarily many solutions, SIAM J. Math. Anal. 17 (1986), 761-771.

[32] B. RUF and P. N. SRIKANTH, Multiplicity results for superlinear elliptic problems with partial interference with the spectrum, J. Math. Anal. Appl. 118 (1986), 15-23.

[33] B. RUF and P. N. SRIKANTH, Multiplicity results for ODEs with nonlinearities crossing all but a finite number of eigenvalues, Nonlinear Anal. 10 (1986), 174-163.

[34] S. Solimini, Some remarks on the number of solutions of some nonlinear elliptic problems, Ann. Inst. H. Poincaré Anal. Non Lineáire 2 (1985), 143-156.

[35] L. WANG, J. WEI and S. YAN, A Neumann problem with critical exponent in non-convex domains and Lin-Ni's conjecture, Trans. Amer. Math. Soc., to appear.

[36] J. WeI and S. YAN, Lazer-McKenna conjecture: the critical case, J. Funct. Anal. 244 (2007), 639-667.

[37] J. WEI and S. YAN, Infinitely many solutions for the prescribed scalar curvature problem, J. Funct. Anal. 258 (2010), 3048-3081.

[38] S. YAN, Multipeak solutions for a nonlinear Neumann problem in exterior domains, Adv. Differential Equations 7 (2002), 919-950.

Department of Mathematics

The Chinese University of Hong Kong

Shatin, Hong Kong

wei@math.cuhk.edu.hk

Department of Mathematics

The University of New England

Armidale, NSW 2351, Australia

syan@turing.une.edu.au 